\title{
2B4 but not PD-1 blockade improves mortality in septic animals with preexisting malignancy
}

\author{
Ching-wen Chen, ${ }^{1}$ Ming Xue, ${ }^{1,2}$ Wenxiao Zhang, ${ }^{1,3}$ Jianfeng Xie, ${ }^{1,2}$ Craig M. Coopersmith, ${ }^{1,4}$ \\ and Mandy L. Ford ${ }^{1,5}$ \\ 'Department of Surgery, Emory University, Atlanta, Georgia, USA. ${ }^{2}$ Department of Critical Care Medicine, Zhongda \\ Hospital, Southeast University, Nanjing, China. ${ }^{3}$ Department of Critical Care Medicine, People's Hospital of Zhengzhou \\ University and Henan Provincial People's Hospital, Zhengzhou, China. ${ }^{4}$ Emory Center for Critical Care, Atlanta, Georgia, \\ USA. ${ }^{5}$ Emory Transplant Center, Atlanta, Georgia, USA.
}

In addition to its well-known beneficial effects for the treatment of several types of cancer, PD-1 blockade has shown encouraging results in preclinical models of sepsis and in a recent clinical trial in sepsis. Because cancer is the most common comorbidity in septic patients, here we aimed to determine the efficacy of PD-1 checkpoint blockade in the setting of sepsis complicated with preexisting malignancy. In a model of established lung cancer followed by cecal ligation and puncture-induced (CLP-induced) sepsis, PD-1 blockade exhibited no therapeutic effect on sepsis survival. This diminished efficacy of PD-1 blockade in cancer septic animals (septic animals with cancer) was characterized by a reduction in both the quality and quantity of PD-1+ responder cells. Specifically, $\mathrm{CD}^{+} \mathrm{T}$ cells isolated from cancer septic animals exhibited decreased CD28 expression and a reduction in the CXCR5 ${ }^{+} \mathrm{PD}-1^{+}$subset. In addition, flow cytometric analysis of T cells isolated from cancer septic animals revealed 2B4 as another possible checkpoint under these conditions. Administration of anti-2B4 to cancer septic animals significantly improved sepsis survival and was associated with increased $\mathrm{T}$ cell costimulatory receptor expression and decreased coinhibitory receptor expression. These results illustrate functions of coinhibitory receptors in the setting of sepsis complicated with cancer.

Authorship note: CMC and MLF contributed equally to this work.

Conflict of interest: The authors have declared that no conflict of interest exists.

Copyright: (c) 2019, American Society for Clinical Investigation.

Submitted: January 31, 2019

Accepted: October 10, 2019

Published: November 14, 2019.

Reference information: JCI Insight.

2019;4(22):e127867.

https://doi.org/10.1172/jci.

insight.127867.

\section{Introduction}

Every year, sepsis develops in more than 1.7 million patients in the United States (1-3). The most common comorbidity in septic patients is cancer, present in 19\% of septic patients, resulting in about 200,000 cancer septic patients (septic patients with cancer) per year $(1,4)$. In patients with cancer, the relative risk for the development of sepsis is 9.77 , and when compared with noncancer patients, the relative risk to be hospitalized with sepsis is $2.77(5,6)$. Among those cancer septic patients, lung cancer is the most prevalent type of cancer, seen in $18 \%$ of patients $(5,7)$. Importantly, the in-hospital mortality rate was significantly higher for cancer septic patients compared with noncancer patients $(37.8 \%$ vs. $24.9 \%)(5,8,9)$.

The immune responses to cancer and sepsis share several similarities, including the induction of $\mathrm{T}$ cell exhaustion. In the tumor microenvironment, continual exposure to tumor antigens leads to $\mathrm{T}$ cell exhaustion, including upregulation of coinhibitory receptors and decreased cytokine production and cytotoxicity (1012). In addition, this deficiency in the adaptive immune system functions systemically, resulting in increased expression of coinhibitory molecules on $\mathrm{T}$ cells in the circulation, inadequate response to vaccination, and an increase in opportunistic infections (13-15). Although the mechanisms leading to $\mathrm{T}$ cell exhaustion during sepsis remain unknown, the phenotypes of $\mathrm{T}$ cell exhaustion are well characterized (16). Postmortem analysis of septic patients and a plethora of animal studies on sepsis revealed that a reduced number of $\mathrm{T}$ cells, increased expression of coinhibitory receptors, and decreased cytokine production were observed in sepsis (17). Given this evidence of immunosuppression during sepsis, several checkpoint blockade therapies have been investigated in different animal sepsis models. PD-1/PD-L1 blockade has also entered sepsis clinical trials with encouraging results (18). For animal studies, administration of anti-PD-1 and anti-PD-L1 both resulted in significantly improved cecal ligation and puncture (CLP) survival $(19,20)$. Blockade of PD-1 also increased 
the survival in the second-hit sepsis model (CLP followed by Candida infection) (21). Mechanistically, PD-1/ PD-L1 blockade lead to decreased cell apoptosis during sepsis, increased Bcl-xL expression on T cells, and enhanced bacterial clearance $(19,20)$. Other coinhibitory molecules, such as CTLA-4, BTLA, and 2B4, also exhibited some therapeutic effect during sepsis. CTLA-4 blockade also resulted in increased survival in the CLP model and decreased cell apoptosis in splenocytes $(21,22)$. Anti-BTLA antibody treatment in a model of dual insults with hemorrhagic shock and sepsis resulted in enhanced recruitment and functionality of innate immune cells; however, anti-BTLA reduced overall survival in this specific sepsis model $(23,24)$. Administration of anti-2B4 antibody increased CLP survival, and studies on 2B4-deficient animals demonstrated the protection is $\mathrm{CD}^{+} \mathrm{T}$ cell-dependent (25). Taken together, these studies highlighted the potential therapeutic implications of checkpoint blockade during sepsis.

Efficacy of PD-1/PD-L1 blockade has been shown to be dependent on several mechanisms in both models of cancer and chronic virus infection. First, CD28 expression on T cells is vital for PD-1 therapy. Blockade of B7 signaling or conditional-KO) of CD28 on CD8 T cells results in ineffective tumor control and inadequate $\mathrm{T}$ cell response after $\mathrm{PD}-1$ therapy. Moreover, the proliferating $\mathrm{CD}{ }^{+}$cells from patients with cancer receiving PD-1 therapy are predominately CD28 $8^{+}$cells $(26,27)$. Second, CXCR5 ${ }^{+} \mathrm{PD}-1^{+}$population provides the proliferative burst after PD-1 therapy. During chronic virus infection, CXCR5 $5^{+} \mathrm{PD}-1^{+}$ cells are found in lymphoid tissues and express higher costimulatory receptors and memory-related transcription factors. After PD-1 blockade, $\mathrm{CXCR} 5^{+}$populations are capable of expanding and differentiating into CXCR5 ${ }^{-}$cells to mediate tumor control (28). Importantly, CXCR5 ${ }^{+} \mathrm{CD}^{+} \mathrm{T}$ cells with high functionality have been found in both circulation and tumor microenvironment on human pancreatic cancer and lung cancer $(29,30)$. Third, host PD-L1 expression is crucial for PD-1 therapy. By using PD-L1 ${ }^{-/-}$mice, 2 groups have shown that PD-L1 expression on host antigen-presenting cells (APCs) but not tumor cells determines the efficacy of PD-1 blockade therapy $(31,32)$. In addition, in patients with metastatic melanoma and ovarian carcinoma treated with checkpoint blockade therapies, patients with clinical response exhibited significantly higher PD-L1 expressed on host APCs compared with the nonresponders (31).

Here, we thought to determine the efficacy of PD-1 checkpoint blockade in a cancer sepsis model. In this study, we found that anti-PD-1 blockade failed to impact sepsis mortality in animals with preexisting cancer and was associated with a reduced number of $C X C R 5^{+} \mathrm{PD}-1^{+}$cells and lower $\mathrm{CD} 28$ expression on $\mathrm{PD}-1^{+}$cells. Conversely, anti-2B4 blockade conferred improved sepsis survival in animals with cancer. This study highlighted the differential impact of PD-1 checkpoint blockade in cancer septic animals and revealed the nonredundant roles of 2 individual checkpoint inhibitors in the setting of cancer and sepsis.

\section{Results}

Anti-PD-1 administration failed to exert a therapeutic effect on cancer-bearing animals during sepsis. To determine antiPD-1 efficacy during sepsis in cancer-bearing animals, we first tested the delayed anti-PD-1 administration protocol described by Brahmamdam et al. (19). In brief, LLC1 tumor cells were subcutaneously implanted in the right thigh of 8-12-week-old C57BL/6J mice. After 3 weeks of tumor growth, tumor-bearing mice or previously healthy $(\mathrm{PH})$ controls were subjected to CLP for sepsis induction. Anti-PD-1 antagonistic mAb (clone RMP1-14) was then given at 48 hours and 72 hours after CLP. In contrast to its known ability to improve sepsis survival in $\mathrm{PH}$ animals (Figure 1A), PD-1 blockade treatment failed to improve CLP survival in animals with cancer: $57 \%$ (11 of 19) of isotype-treated animals survived, whereas only $37 \%$ (7 of 19) of anti-PD-1 treated animals survived (Figure 1B). It is known that PD-1 blockade improves sepsis survival by decreasing lymphocyte apoptosis and increasing anti-apoptotic protein expression. To further evaluate anti-PD-1 efficacy on a cellular level, we assessed caspase $3 / 7$ activity and anti-apoptotic protein $\mathrm{Bcl}-\mathrm{xL}$ expression on $\mathrm{CD} 4^{+}$and $\mathrm{CD}^{+} \mathrm{T}$ cells isolated from both $\mathrm{PH}$ animals and those with cancer. In $\mathrm{PH}$ animals, anti-PD-1 was able to reduce cell apoptosis and increase $\mathrm{Bcl}-\mathrm{xL}$ expression on both $\mathrm{CD}^{+}$and $\mathrm{CD} 8^{+} \mathrm{T}$ cells as previously described (19). However, in animals with cancer, the anti-PD-1 effect was diminished; both caspase $3 / 7$ and Bcl-xL expression were unchanged compared with isotype treatment (Figure 1, C and D). Thus, PD-1 blockade was ineffective at inhibiting lymphocyte apoptosis in cancer septic animals.

We previously demonstrated that T cells isolated from LLC1 animals with cancer displayed higher coinhibitory receptor expression at the baseline compared with $\mathrm{PH}$ animals. It is possible that PD-1 signaling on $\mathrm{T}$ cells was already occurring on T cells prior to the CLP insult. To test the possibility that our dosing strategy was missing the therapeutic window in cancer hosts, we adjusted our treatment protocol from dosing at delayed time points (day 2, day 3 after CLP) to early anti-PD-1 blockade (days 0, 2, 4, and 6 after CLP, Clone 29F.1A12). 

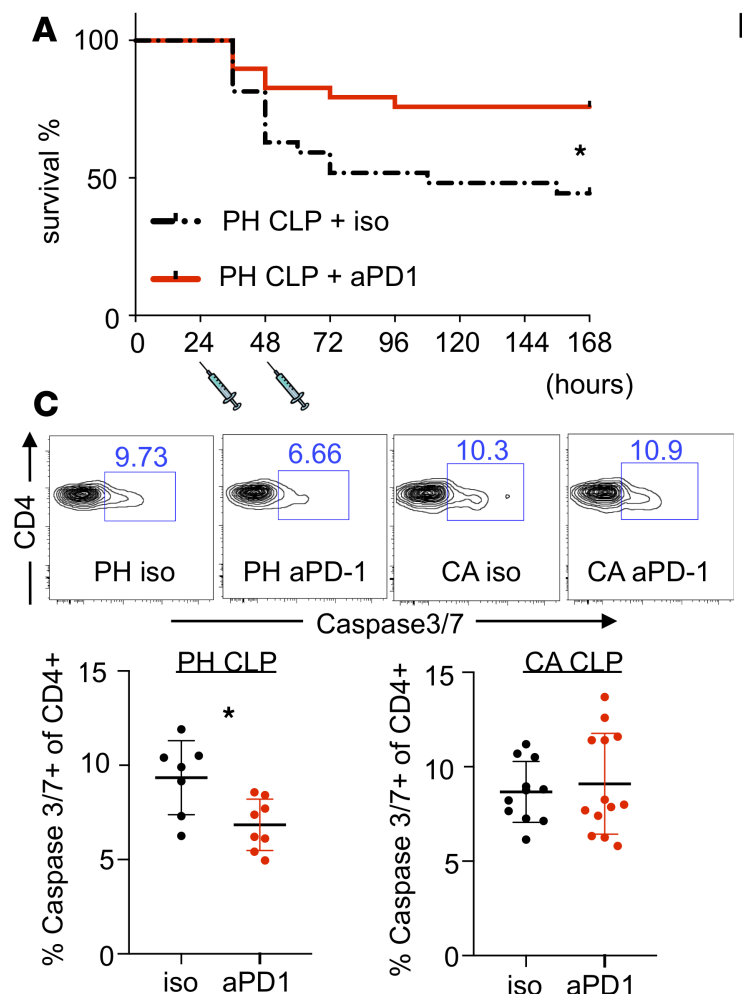

D
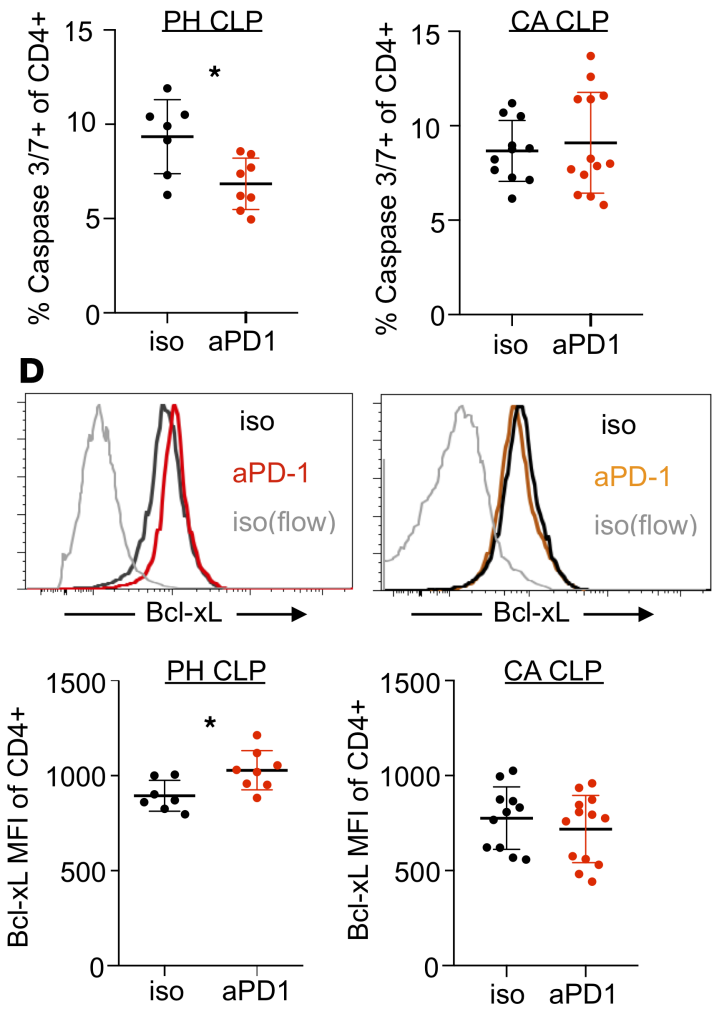
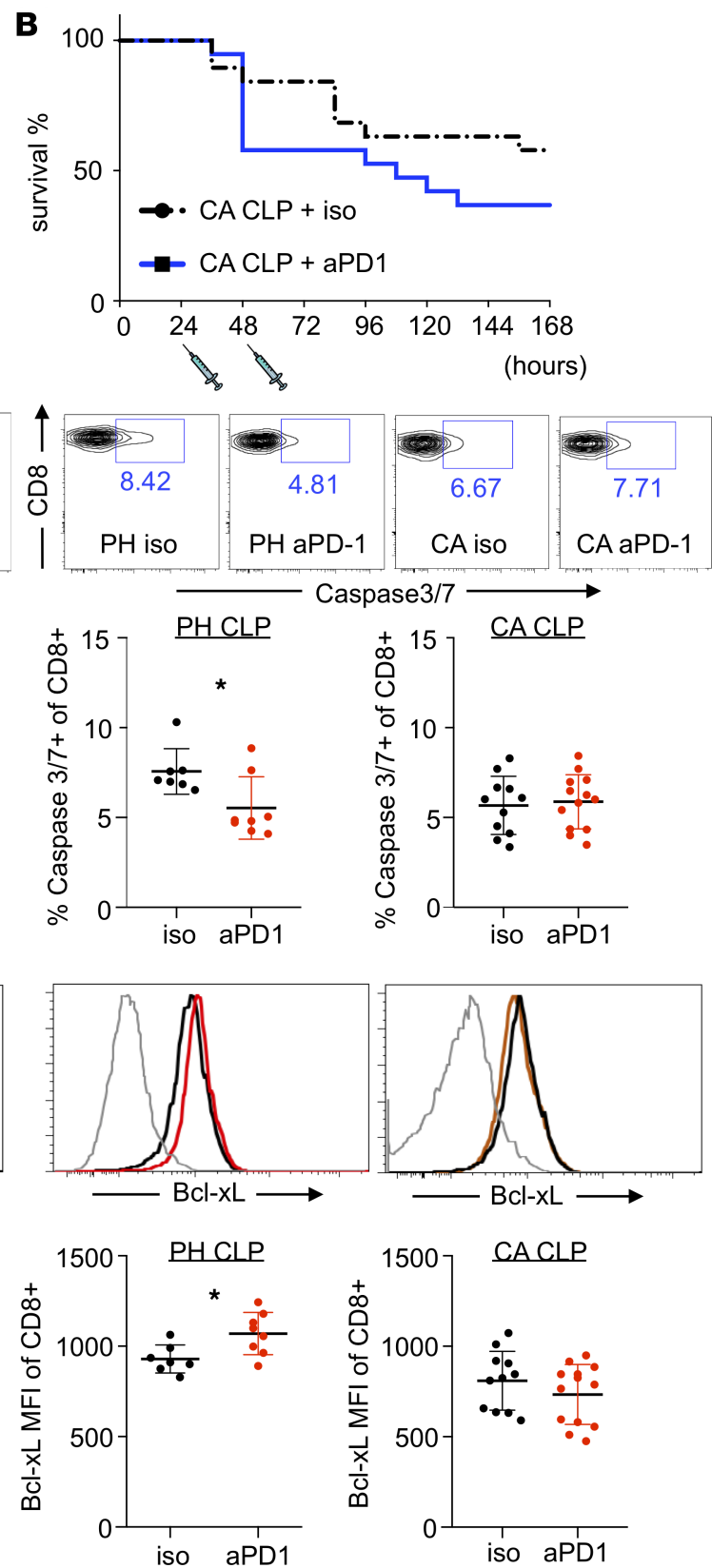

Figure 1. PD-1 blockade fails to improve sepsis survival and has no effect on reversing T cell apoptosis in a cancer sepsis model. (A) PD-1 antagonistic monoclonal antibody (clone RMP1-14, $n=29$ ) or an isotype control antibody $(n=27)$ were administered to previously healthy $(\mathrm{PH})$ animals via i.p. injection at day 1 and day 2 after cecal ligation and puncture (CLP). Animals were followed for 7 days for survival. The log-rank test was performed. (B) LLC tumor cells were subcutaneously injected in the thigh and allowed to grow for 3 weeks. At day 21, animals with cancer (CA) were subjected to CLP surgery to induce sepsis. Cancer septic animals were treated with PD-1 antagonistic monoclonal antibody or isotype control antibody at day 1 and day 2 after CLP. Animals were followed for survival for 7 days. $n=19$ in each group. The log-rank test was performed. (C) Active caspase 3 was assessed in splenic CD4 ${ }^{+}$ and CD8 ${ }^{+}$T cells isolated on day 2 from PH septic animals $(n=7-8)$ or cancer septic animals $(n=11-12)$ treated with either anti-PD-1 or isotype control. (D) Anti-apoptotic protein Bcl-xL was assessed in splenic $\mathrm{CD} 4^{+}$and $\mathrm{CD} 8^{+}$T cells isolated on day 2 from PH septic animals $(n=7-8)$ or cancer septic animals $(n$ = 11-12) treated with either anti-PD-1 or isotype control. Iso (flow) represents the flow cytometry isotype control staining for Bcl-xL staining. The 2-tailed Student's $t$ test was performed. ${ }^{*} P<0.05$.

However, PD-1 blockade still failed to improve survival in cancer septic animals (Supplemental Figure 1; supplemental material available online with this article; https://doi.org/10.1172/jci.insight.127867DS1).

Both PD-1 and PD-L1 are highly expressed during cancer sepsis. We next sought to determine the mechanisms underlying the ineffectiveness of PD-1 blockade in the setting of cancer and sepsis. We first confirmed adequate expression of both the receptor and ligand in the setting of cancer sepsis. In PH animals, 
A
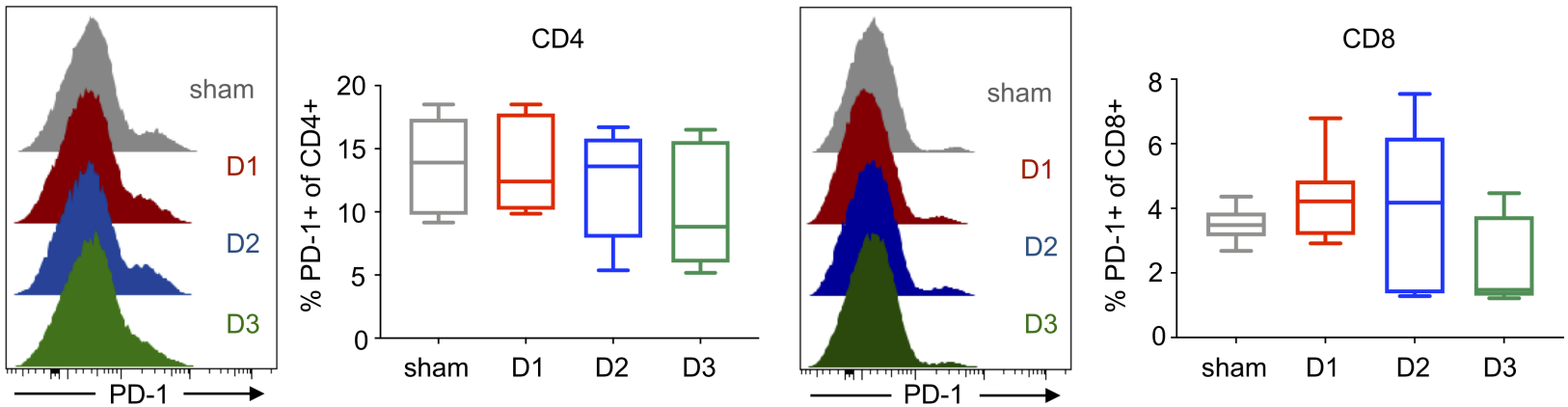

B
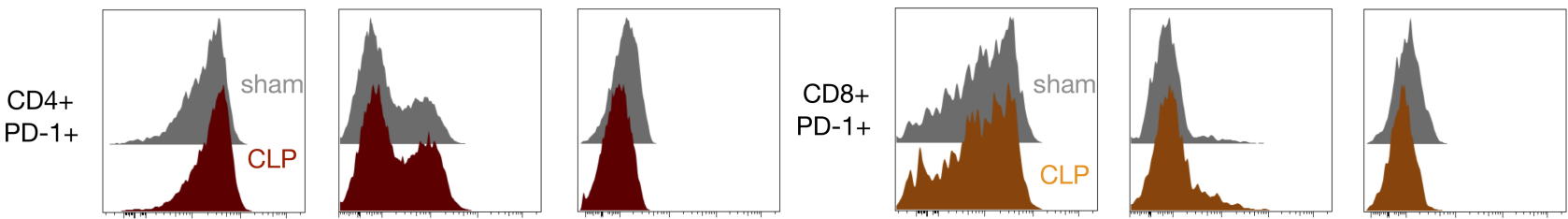

$\mathrm{CD} 44$

CD69

CD28
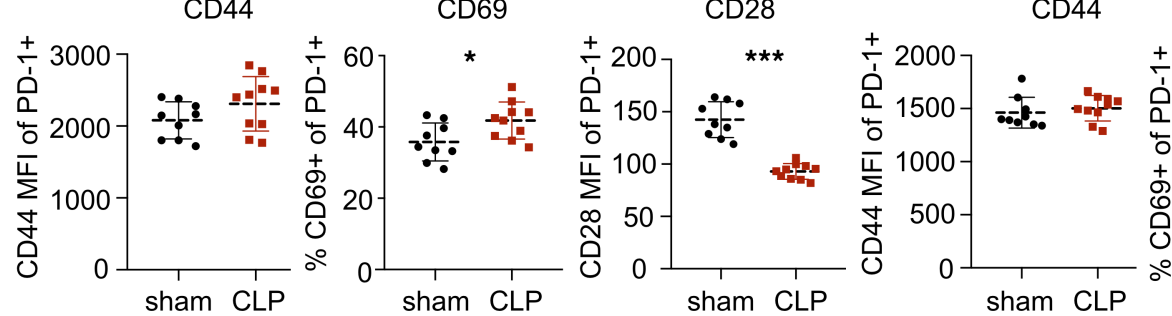

CD69

CD28
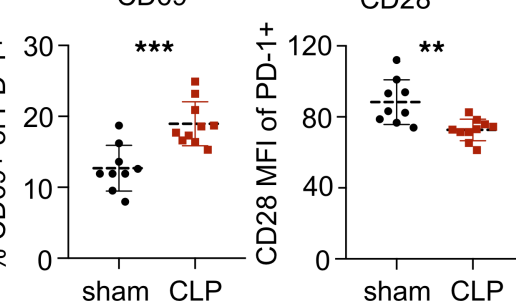

Figure 2. PD-1 expression is maintained during sepsis in animals with cancer, but PD-1+ cells exhibit dysregulated phenotypes during sepsis. (A) Cancer septic or sham animals were sacrificed at indicated time points. Spleens were harvested, and PD-1 expression was determined $(n=6-8)$. The 1-way ANOVA test was performed. (B) CD44, CD69, and CD28 expression was determined on CD4+PD-1+ cells and CD8 ${ }^{+} P D-1^{+}$cells at 24 hours after cecal ligation and puncture (CLP). Cancer sham animals were defined as a control group. $n=9$-14/group. The 2-tailed Student's $t$ test was performed. ${ }^{*} P<0.05,{ }^{* *} P<0.01,{ }^{* * *} P<0.001$.

the frequency of PD-1-expressing T cells was significantly increased after the CLP insult $(19,20)$. However, in cancer septic animals, frequencies of $\mathrm{PD}-1$ expressing both $\mathrm{CD} 4^{+}$and $\mathrm{CD} 8^{+} \mathrm{T}$ cells were maintained at the same levels as sham surgery controls from day 1 to day 3 after CLP (Figure 2A). We also tested the PD-L1 expression on host APCs to confirm whether cancer septic APCs could promote negative signaling through the PD-1/PD-L1 pathway. Results showed that dendritic cells, macrophages, and MDSC-like cells in the spleen all strongly upregulated PD-L1 expression after sepsis (Supplemental Figure 2).

Cancer sepsis is associated with reduced CD28 expression and lower frequencies of CXCR $5^{+} P D-1^{+}$stem cell-like $C D 8^{+} T$ cells. Several recent publications have defined the mechanisms by which PD-1 blockade works in models of chronic viral infection. First, Kamphorst et al. showed that CD28 expression is absolutely required for $\mathrm{PD}-1$ blockade to rescue $\mathrm{CD} 8^{+} \mathrm{T}$ cell responses (27). Thus, we queried whether $\mathrm{CD} 28$ expression was reduced during sepsis. At 24 hours after CLP, CD4 $4^{+} \mathrm{PD}-1^{+}$and $\mathrm{CD} 8^{+} \mathrm{PD}-1^{+}$from sham cancer animals and animals with cancer were stained with a series of markers (Figure 2B). For activation markers on $\mathrm{CD} 4^{+} \mathrm{PD}-1^{+}$cells, although CD44 expression was similar between sham and CLP groups, the percentage of $\mathrm{CD}_{69^{+}}$cells was significantly increased in the CLP group, indicating that cells in the PD- $1^{+}$population became activated during CLP. Interestingly, we found that CD28 expression was significantly reduced on septic CD4 $4^{+} \mathrm{PD}-1^{+} \mathrm{T}$ cells (Figure $2 \mathrm{~B}$ ). For $\mathrm{CD} 8^{+} \mathrm{PD}-1^{+}$cells, an increased percentage of $\mathrm{CD} 69^{+}$cells were also observed. Of note, $\mathrm{CD} 8^{+} \mathrm{PD}-1^{+}$cells dramatically lost $\mathrm{CD} 28$ expression during sepsis, similar to $\mathrm{CD} 4^{+} \mathrm{PD}-1^{+}$cells (Figure $2 \mathrm{~B}$ ). Thus, compromised $\mathrm{CD} 28$ expression on both $\mathrm{CD} 4^{+}$and $\mathrm{CD} 8^{+} \mathrm{T}$ cells may underlie the reduced efficacy of PD-1 blockade during cancer sepsis.

Im et al. recently showed that CXCR5 ${ }^{+} \mathrm{PD}-1^{+} \mathrm{CD} 8^{+} \mathrm{T}$ cells exhibit self-renewal potential and the ability to reconstitute the response following PD-1 blockade in the setting of chronic viral infection. CXCR5 ${ }^{+}$ PD- $1^{+}$responder cells are predominantly TIM- $3^{-}$and $2 \mathrm{~B}^{-}(28)$. We thus tested the hypothesis that PD-1 

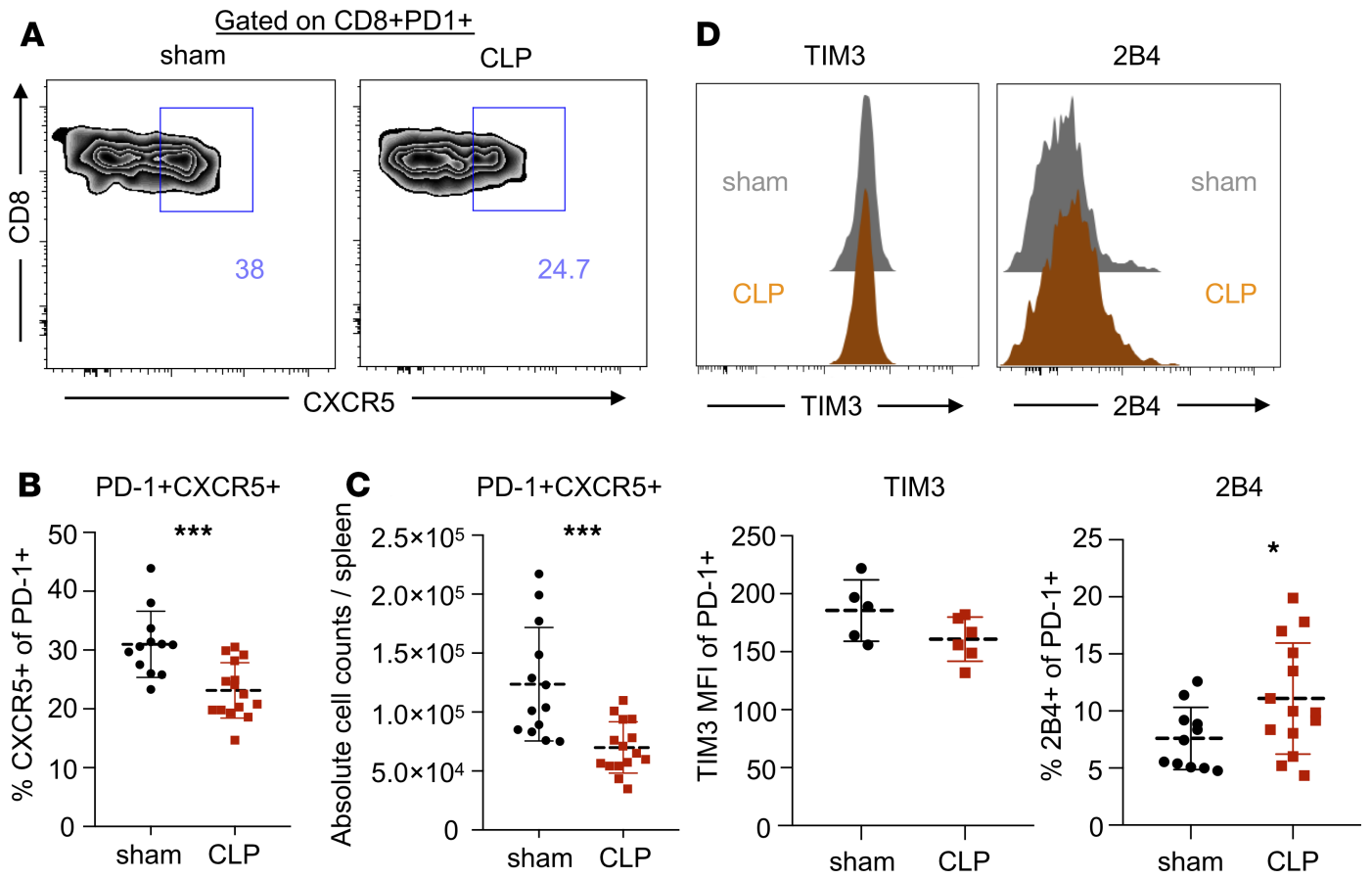

Figure 3. CXCR5+PD-1+CD8 ${ }^{+}$cells decrease significantly after sepsis. Animals with cancer animals were subjected to cecal ligation and puncture (CLP) and sacrificed at 24 hours after CLP. Splenic CXCR5 ${ }^{+} P D-1^{+} C D 8^{+}$cells were analyzed. (A) Representative flow plots from cancer sham and cancer CLP depicting CXCR5 staining on CD8 ${ }^{+}$PD-1 $1^{+}$T cells. (B) Summary data showing frequency of CXCR5 $5^{+}$cells among CD8+PD-1+ cells. (C) Summary plot of the absolute number of CXCR5 ${ }^{+}$PD- $1^{+}$CD8 $8^{+}$cells in the spleen. $n=13-15$ /group. (D) Representative flow plots and summary plots of TIM-3 and 2B4 expression on PD-1+CD8 ${ }^{+}$ cells. $n=5$-14/group. The 2-tailed Student's $t$ test was performed. ${ }^{*} P<0.05,{ }^{* *} P<0.001$.

blockade may be ineffective during cancer sepsis because of a dearth in this critical population of CXCR $5^{+}$ $\mathrm{PD}-1^{+} \mathrm{CD} 8^{+} \mathrm{T}$ cells. Interestingly, we observed that the percentage of $\mathrm{CXCR} 5^{+}$of $\mathrm{PD}-1^{+} \mathrm{CD} 8^{+}$cells in splenocytes was significantly declined in CLP animals at 24 hours after surgery compared with the sham group (Figure 3, A and B). In addition, the absolute cell counts of CXCR5 ${ }^{+} \mathrm{PD}-1^{+} \mathrm{CD} 8^{+}$in splenocytes also was significantly decreased in CLP animals at 24 hours after CLP (Figure 3C). Analysis of coinhibitory molecule expression on PD-1+ $1^{+} \mathrm{CD} 8^{+} \mathrm{T}$ cells showed no difference in TIM-3 expression but a significant increase in the frequency of $2 \mathrm{~B} 4+$ cells within this population in cancer septic animals compared with cancer sham animals (Figure 3D), suggesting that animals with cancer are losing a subset of the PD-1 blockade "responder" cell populations during sepsis.

SPADE and CITRUS analysis revealed unique $C D 8^{+} T$ cell populations in cancer septic animals. Given these results suggesting that the factors critical to the effectiveness of PD-1 blockade are lacking during cancer sepsis, we sought to identify additional coinhibitory signals that might be effectively targeted to control dysregulated $\mathrm{T}$ cell responses during sepsis. Both $\mathrm{PH}$ animals and those with cancer received CLP and were sacrificed at 24 hours after surgery. Splenocytes were harvested and stained with CD44, 2B4, PD-1, BTLA, and LAG-3. CD ${ }^{+} \mathrm{CD}^{+}$cells were gated in FlowJo and exported to Cytobank to perform algorithm analysis. These algorithms provided an unbiased and comprehensive analysis rather than traditional 2-dimensional and hierarchical analysis using FlowJo. First, Spanning-tree Progression Analysis of Density-normalized Events (SPADE) was performed, and use of 50 nodes conferred better resolution in our model. After adjustments, multiple $t$ test comparisons identified 2 distinct clusters as being significantly elevated in cancer septic animals compared with PH septic animals (Figure 4, A and B). The first cluster, located on the right side of the SPADE tree-like figure, contained cells expressing a

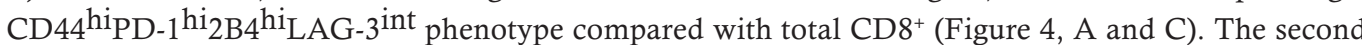
cluster upregulated in cancer septic animals contained cells possessing a CD $44^{\mathrm{hi}} \mathrm{PD}-1{ }^{1 \mathrm{o}} 2 \mathrm{~B} 44^{\mathrm{hi}} \mathrm{LAG}-3^{\mathrm{int}}$ phenotype (Figure 4, A and C).

Next, the CITRUS (cluster identification, characterization, and regression) algorithm was performed on the same data sets to confirm the SPADE findings. CITRUS is a specific algorithm for fully automated discovery of statistically significant differences between groups. By choosing Nearest Shrunken Centroid 
A

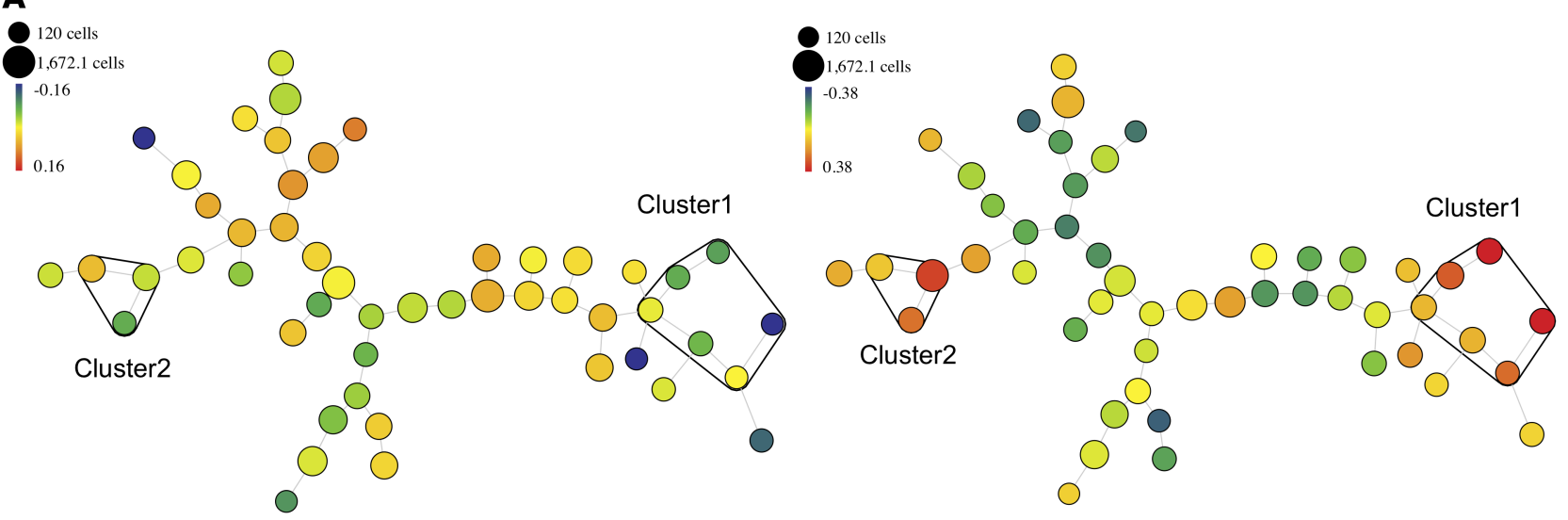

B

C - Cluster1 - Cluster2 - Total CD8

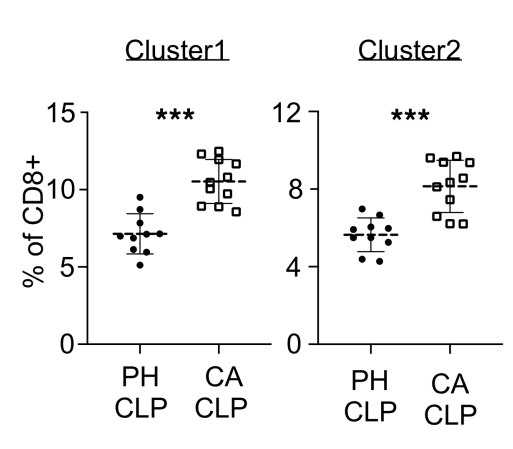

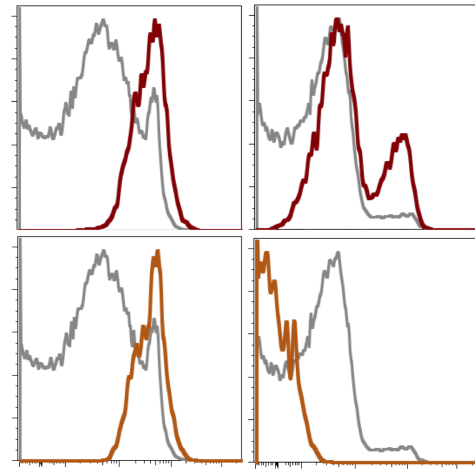

CD44

PD-1

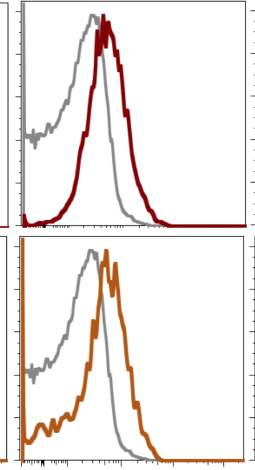

2B4

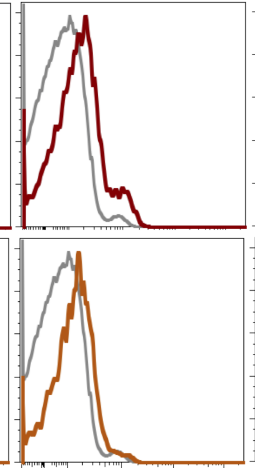

BTLA

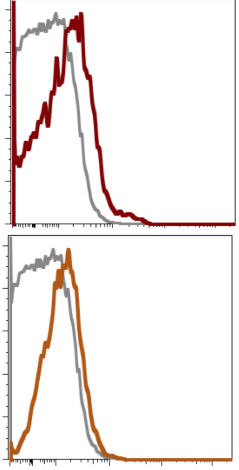

LAG-3

Figure 4. SPADE algorithm identifies $\mathbf{2} \mathrm{CD8}^{+} \mathbf{T}$ cell clusters that are upregulated in cancer septic animals. Previously healthy (PH) animals and animals with cancer (CA) were subjected to cecal ligation and puncture (CLP) and euthanized at 24 hours after CLP. Splenocytes were stained with CD3, CD8, CD44, PD-1, 2B4, BTLA, and LAG-3. CD3+CD8+ T cells were pregated in Flowjo and exported to Cytobank. SPADE analysis was performed using the 50 node setting. PH animals are defined as a baseline to benefit group comparisons. (A) Representative SPADE tree figure generated by the algorithm. Tree figure (top row left) represents PH septic animals, and tree figure (top row right) represents cancer septic animals. The clusters were discovered by multiple $t$ tests with adjustment. Only the clusters upregulated in animals with cancer are shown here. (B) Summary plot of percentage of 2 clusters among total CD8 ${ }^{+} T$ cells. (C) Phenotype histograms of 2 clusters. The gray line represents total CD8 ${ }^{+}$T cells from PH CLP mice, serving as a baseline. $n=10-11$. The multiple $t$ test was performed to identify significantly changed nodes. ${ }^{* *} P<0.0001$

(PAMR) association model and minimum FDR in CITRUS, the algorithm identified 3 populations that were changed between cancer septic animals and $\mathrm{PH}$ septic animals (Figure 5A). The summary plots and CITRUS plot showed 2 groups of cells were increased in cancer septic animals: 1) Node-209985; and 2) Node-209991 and 209995 (clustered together) (Figure 5, A and B). The phenotype of cells in the first node 209985 was CD44 ${ }^{\text {hi PD- }} 1^{1 \mathrm{l}_{2}}$ B $4{ }^{\text {hi }}$ LAG-3 ${ }^{\text {int }}$, and the phenotype of cells in the second group 209991 and 209995 was CD44hi ${ }^{\text {hi }}-1^{\text {hi }} 2$ B $4^{\text {hi }}$ LAG-3 ${ }^{\text {int }}$ (Figure 5C and Supplemental Figure 3). Notably, these results were consistent with our SPADE data. We also performed CITRUS on CD4 ${ }^{+} \mathrm{T}$ cells and observed that similar populations $\left(\mathrm{CD} 44^{\mathrm{hi}} \mathrm{PD}-1^{\mathrm{lo}} 2 \mathrm{~B} 4^{\mathrm{hi}}\right.$ and $\left.\mathrm{CD} 44^{\mathrm{hi}} \mathrm{PD}-1^{\mathrm{hi}} 2 \mathrm{~B} 4^{\mathrm{hi}}\right)$ are increased in cancer septic animals (Supplemental Figure 4).

Finally, to further confirm this result, traditional flow analysis was performed by manual gating on $2 \mathrm{~B} 4$ and $\mathrm{PD}-1$ (Figure 6A). Both $2 \mathrm{~B} 4^{+} \mathrm{PD}-1^{-}$and $\mathrm{PD}-1^{+} 2 \mathrm{~B} 4^{-}$in $\mathrm{CD}^{+} \mathrm{T}$ cells were increased in cancer septic animals compared with $\mathrm{PH}$ septic animals (Figure $6 \mathrm{~B}$ ). Thus, the data suggest that $2 \mathrm{~B} 4$ is expressed and potentially functioning on both overlapping and unique populations relative to PD- 1 in cancer septic animals. Because anti-PD-1 was ineffective at improving sepsis survival in the setting of cancer sepsis, we shifted our interest toward blockade of 2B4 signaling in cancer septic animals.

Blockade of 2B4 improves survival in cancer septic animals. To determine the timing of $2 \mathrm{~B} 4$ blockade, we first assessed 2B4 expression kinetics on T cells in cancer septic animals. The frequencies of 2B4-expressing $\mathrm{CD}^{+}$and $\mathrm{CD}^{+} \mathrm{T}$ cells were significantly increased at day 1 and day 2 after CLP and then 

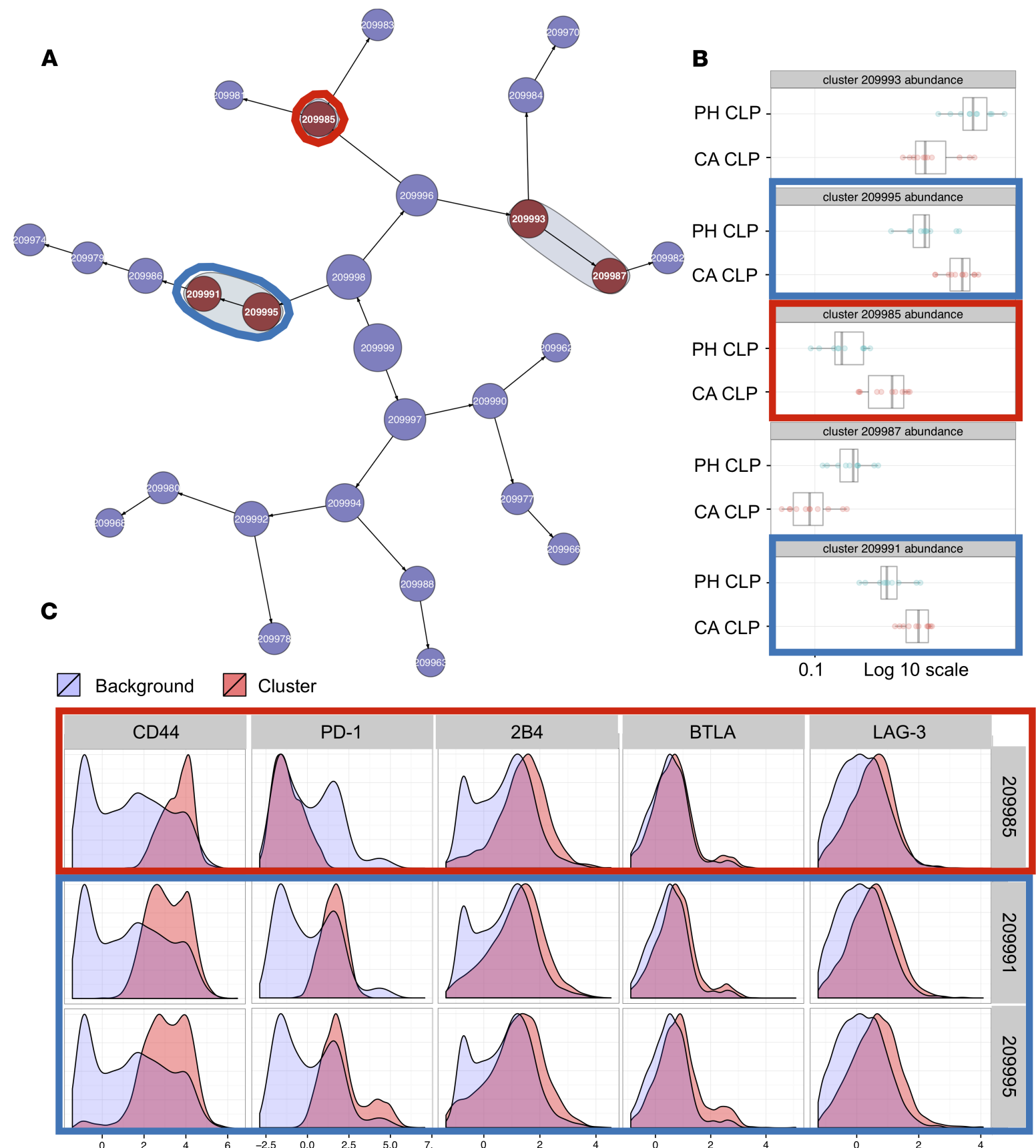

Figure 5. CITRUS identifies similar CD8 ${ }^{+}$populations that are elevated in cancer septic animals. The CD8 ${ }^{+}$data sets described in Figure 4 were exported into the CITRUS algorithm (Cytobank) and PAMR association model with minimum FDR was selected. (A) Plots of abundance and significantly changed clusters were automatically generated by CITRUS. (B) The abundance summary plot of nodes that were significantly different between previously healthy (PH) cecal ligation and puncture (CLP) and cancer (CA) CLP groups. (C) Phenotype histograms of the nodes that are increased in cancer septic animals. $n=10-11$.

decreased at day 3 after CLP (Figure 7A). Because the kinetics of the increase in 2B4+ T cells was similar to that observed in $\mathrm{PH}$ septic animals, we decided to treat cancer septic animals with anti-2B4 following the previously described protocol (25).

To determine the efficacy of 2B4 blockade in the setting of cancer sepsis, animals were treated with anti-2B4 or isotype after CLP at days $0,2,4$, and 6 . Results indicated that anti-2B4 significantly improved sepsis survival in animals with cancer (39\% survival in anti-2B4-treated vs. $13 \%$ survival in isotype-treated, Figure 7B). To understand the impact of 2B4 blockade on $\mathrm{T}$ cell dysregulation during cancer sepsis, cancer septic animals received 1 dose of anti-2B4 treatment and were sacrificed at 24 hours after CLP. One mechanism that leads to $\mathrm{T}$ cell dysfunction during sepsis is the loss of costimulatory molecules and 
A

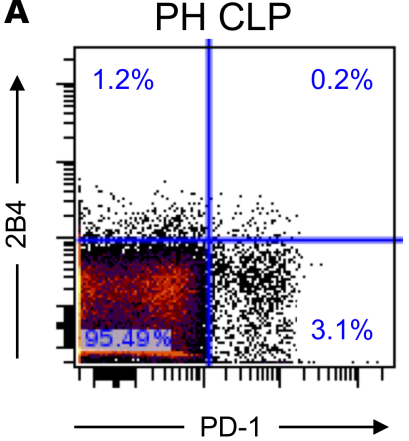

B

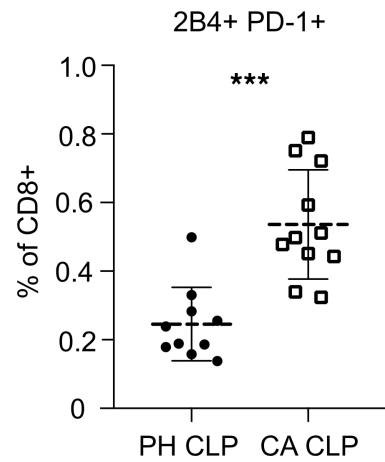

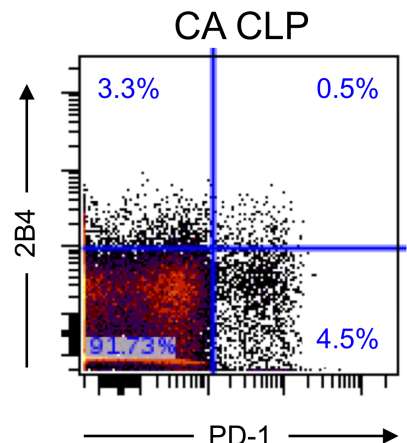

2B4- PD-1+

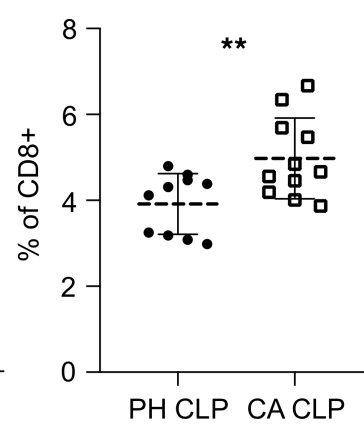

2B4+ PD-1-

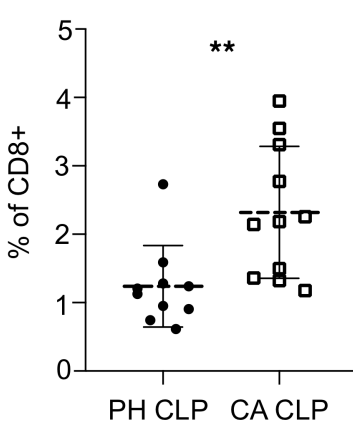

2B4- PD-1-

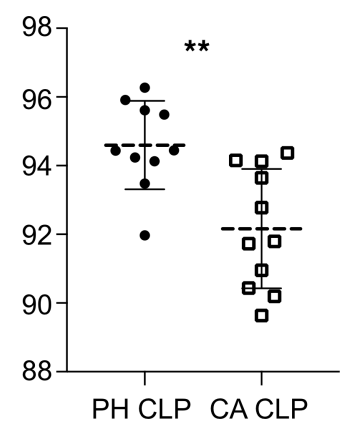

Figure 6. Traditional Flowjo gating strategy confirms the findings from SPADE and CITRUS. Using traditional Flowjo gating strategy, PD-1 and 2B4 coexpressing or single expressing cells were analyzed among $\mathrm{CD}^{+} \mathrm{T}$ cells. (A) Representative flow plots for PD-1 and $2 \mathrm{~B} 4$ expression in previously healthy (PH) cecal ligation and puncture (CLP) and cancer (CA) CLP animals. (B) Summary plots of different populations among total CD8 ${ }^{+}$cells. $n=10-11$. The 2-tailed Student's $t$ test was performed. ${ }^{* *} P<0.01,{ }^{* *} P<0.001$.

increased coinhibitory molecules on T cells. Therefore, we assessed the costimulatory receptor expression on $\mathrm{CD}^{+} \mathrm{T}$ cells and found that anti-2B4 treatment significantly increased CD48 expression (Figure 8A), which may provide adjacent $\mathrm{T}$ cells with stronger CD2 costimulation signals during sepsis. No change in CD28 expression was observed, but significantly lower ICOS expression on CD4 ${ }^{+} \mathrm{T}$ cells in anti-2B4treated animals was noted (Figure $8 \mathrm{~A}$ ). The expression of CD48 was significantly elevated, and CD28 exhibited a trend toward increased expression after 2B4 blockade on $\mathrm{CD}^{+} \mathrm{T}$ cells during sepsis (Fig ure 8B). Next, coinhibitory receptor expression on $\mathrm{T}$ cells after treatments was determined. Interestingly, anti-2B4 treatment significantly decreased PD-1 expression on $\mathrm{CD}^{+} \mathrm{T}$ cells but not $\mathrm{CD} 8^{+} \mathrm{T}$ cells and significantly reduced TIGIT expression on $\mathrm{CD} 8^{+} \mathrm{T}$ cells but not on $\mathrm{CD} 4^{+} \mathrm{T}$ cells (Figure $9, \mathrm{~A}$ and $\mathrm{B}$ ). Strikingly, we found that anti-2B4 treatment significantly reduced the frequencies of CTLA-4-expressing cells among both $\mathrm{CD}^{+}$and $\mathrm{CD}^{+} \mathrm{T}$ cell populations (Figure 9, $\mathrm{A}$ and $\mathrm{B}$ ). In addition, intracellular cytokine staining following ex vivo restimulation revealed that anti-2B4-treated animals exhibited increased frequencies of TNF- and IL-2-producing cells within the $\mathrm{CD}^{+} \mathrm{T}$ cell compartment (Figure 10A) and increased frequencies of IFN- $\gamma-$, TNF-, and IL-2-producing cells among CD8 ${ }^{+} \mathrm{T}$ cells (Figure 10B) compared with cells isolated from isotype-treated animals during sepsis. These data indicated that anti-2B4 treatment of cancer septic animals improved $\mathrm{T}$ cell effector function.

Interestingly, anti-2B4 treatment significantly reduced both the frequency of Foxp3 ${ }^{+}$among CD $4^{+}$ cells and the MFI of Foxp3 within that subset (Figure 11, A-C). The decrease in Foxp3 expression in Tregs after 2B4 blockade (Figure 11D) may have indicated a loss of suppressive function and/or higher Treg plasticity following 2B4 blockade. Thus, we next examined GITR and CTLA-4 expression in Tregs, both of which are important for Tregs to regulate immune responses. We found no difference in GITR expression, but we also observed that CTLA-4 expression in Foxp $3^{+}$cells was significantly decreased after 2B4 blockade, suggesting Treg in 2B4 blockade group may exhibit lower suppressive activity (Figure 11E). We also confirmed that CTLA-4 expression was significantly reduced in the $\mathrm{T}$ conventional population (Figure 11F). 
A

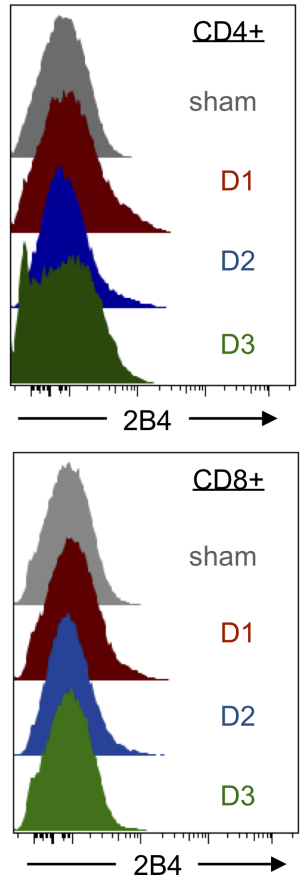

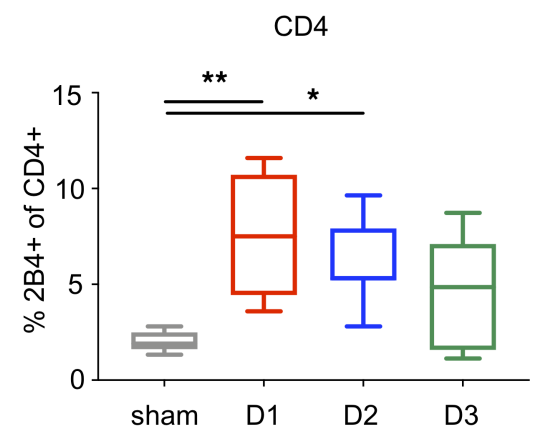

CD8

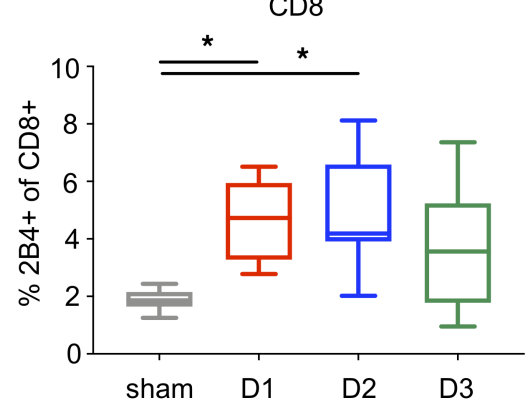

Figure 7. 2B4 is increased on $\mathrm{T}$ cells and 2B4 blockade improves sepsis survival in animals with cancer. (A) Cancer septic or sham animals were sacrificed at different time points to determine 2B4 expression on spleen T cells. $n=6-8$. The 1-way ANOVA test was performed. ${ }^{*} P<0.05$, ${ }^{* *} P<0.01$. (B) 2 B4 blockade antibody (clone 2B4) and isotype control antibody were administrated to cancer septic animals by i.p. injection on days $0,2,4$, and 6 after cecal ligation and puncture (CLP). Mice were followed for 7 days for survival. $n=23,24$ in 2 groups, and the log-rank test was performed.

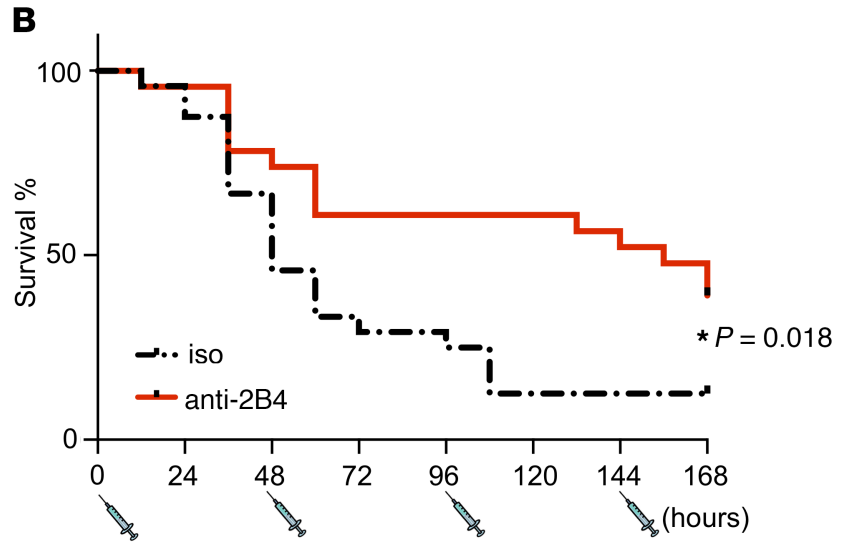

\section{Discussion}

In this study, we demonstrated that anti-PD-1 blockade failed to improve sepsis survival in animals with cancer. This impaired efficacy of PD-1 blockade was associated with a decrease in CD28 expression on $\mathrm{PD}-1^{+}$cells and a loss of $\mathrm{PD}-1^{+} \mathrm{CXCR} 5^{+} \mathrm{CD} 8^{+}$stem cell-like responder cells during sepsis. Multi-parameter flow analysis identified the expression of another coinhibitory receptor, 2B4, as a potential therapeutic target in cancer septic animals. Importantly, we found that blocking 2B4-mediated coinhibitory signals was sufficient to improve sepsis survival in animals with cancer. These results highlighted the importance of personalized therapy in sepsis and highlighted the potential utility of animal models in modeling the physiology of comorbid conditions that may impact sepsis pathophysiology.

Altered sepsis pathophysiology has been previously described in hosts with preexisting malignancy. For example, the inhibition of lymphocytes apoptosis is well-known to improve sepsis survival in $\mathrm{PH}$ animals (33-35). However, in animals with preexisting cancer, prevention of lymphocyte apoptosis by overexpression of Bcl-2 or using $\mathrm{Bim}^{-/}$mice lead to increased mortality in a pneumonia sepsis model (36). Moreover, we have previously shown that during sepsis, the dysregulated host response to infection may be exaggerated in animals with cancer compared with $\mathrm{PH}$ animals (37, 38). Significantly increased activation markers (CD25, CD69) and coinhibitory receptors (PD-1, 2B4) expression were found on both $\mathrm{CD}^{+}$and $\mathrm{CD}^{+} \mathrm{T}$ cells. In addition, increased TNF secretion was observed following ex vivo $\mathrm{T}$ cell restimulation. These results demonstrated that the host 
A

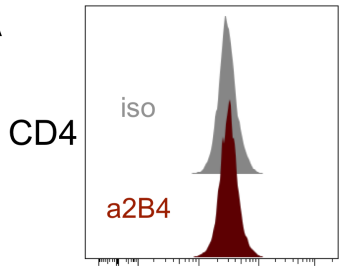

CD2

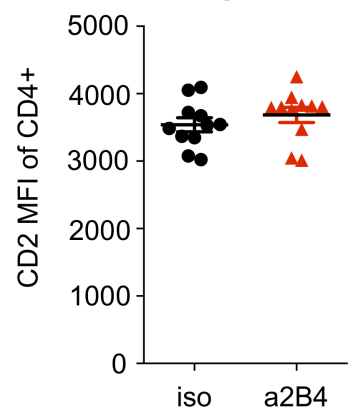

B

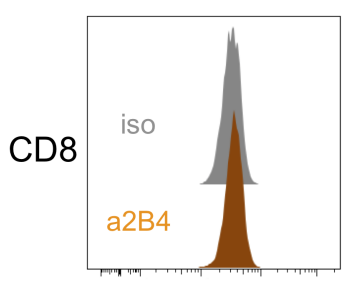

CD2

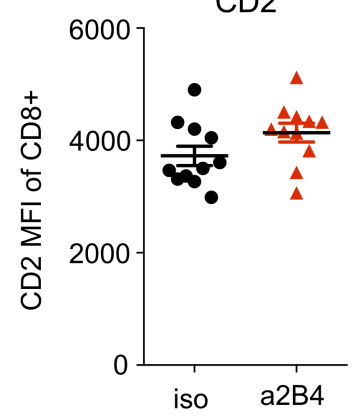

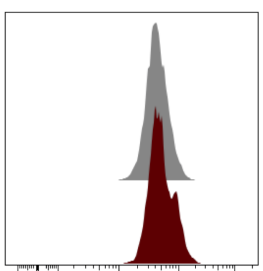

CD48
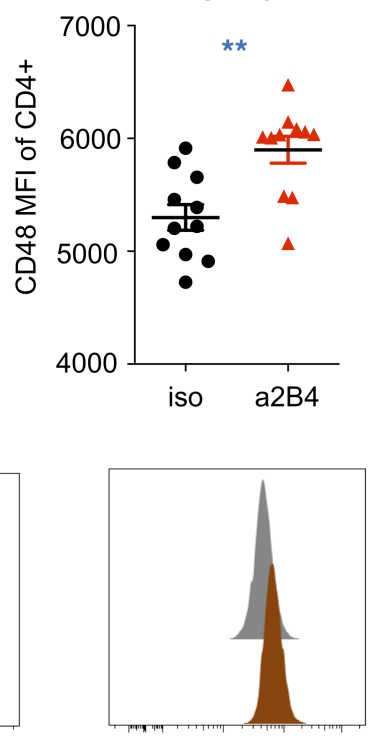

CD48

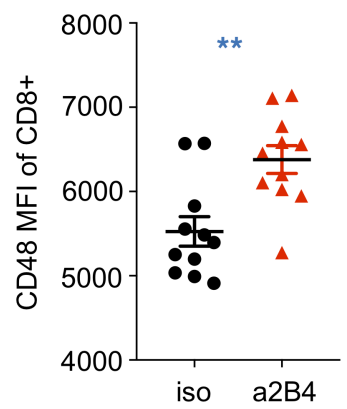

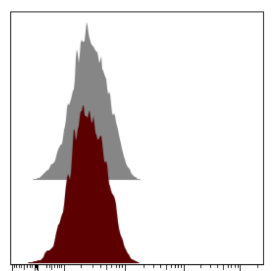

CD28
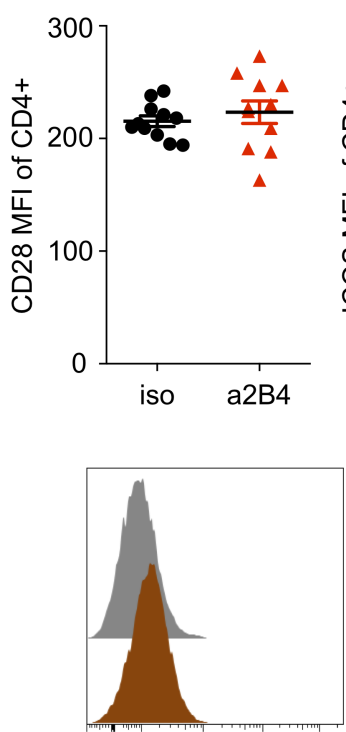

CD28

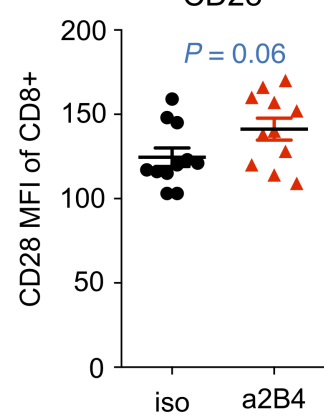

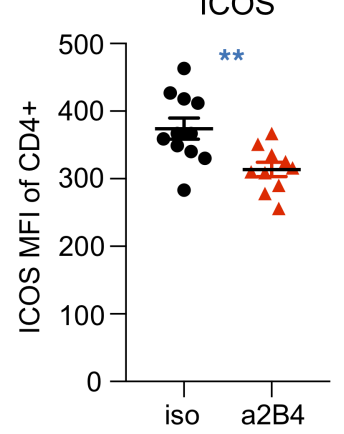

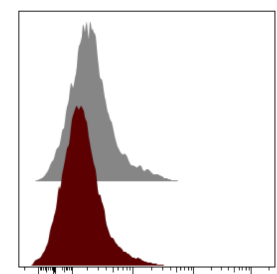

ICOS

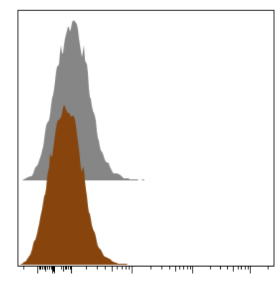

ICOS

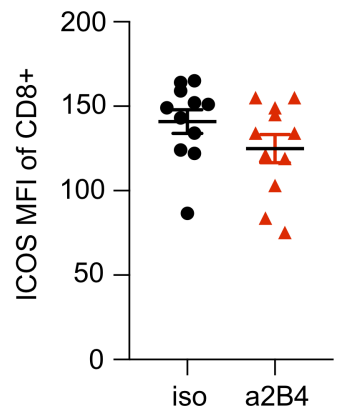

Figure 8. Costimulatory receptors on T cells isolated from cancer septic mice are altered following anti-2B4 treatment. The expression of costimulatory receptors on splenic T cells was assessed following cecal ligation and puncture (CLP) in the presence or absence of 2B4 blockade. Septic animals receiving anti-2B4 or isotype antibody after surgery were sacrificed at 24 hours after CLP. (A) Summary plots of costimulatory receptors on total CD4+ $T$ cells. (B) Summary plots of costimulatory receptors on total CD8 ${ }^{+}$T cells. $n=10-11$ /group. The 2 -tailed Student's $t$ test was performed. ${ }^{*} P<0.05$, ${ }^{*} P<0.01$.

response to sepsis in animals with cancer was characterized by increased activation and inflammation relative to that observed in healthy animals (37). Therefore, blockade of PD-1 may be insufficient to overcome the immune dysregulation in cancer septic animals and potentially may even further amplify the overwhelming inflammation during cancer sepsis.

Other immunologic features known to be required for optimal response to PD-1 blockade were dysregulated in cancer septic hosts. First, Kamphorst et al. (27) found that CD28 signals are absolutely required for the efficacy of PD-1 blockade. CD28 deletion on T cells results in impaired expansion during PD-1 blockade on a virus infection model. In addition, Kamphorst et al. (26) demonstrated that in patients with non-small cell lung cancer treated with PD-1 targeted therapy, the responding PD- $1^{+} \mathrm{Ki}-67^{+}$cells highly expressed CD28 costimulatory molecules. Both sets of results emphasized that $\mathrm{PD}-1^{+} \mathrm{T}$ cells require costimulation signals to respond to PD-1 blockade therapy. In our cancer sepsis model, PD-1 $1^{+}$cells lost CD28 and increased other coinhibitory molecules during sepsis, indicating those $\mathrm{PD}-1^{+} \mathrm{T}$ cells exhibited lower responding capacity to PD-1 blockade therapy. In addition, Im et al. (28) found that CXCR5 ${ }^{+} \mathrm{CD} 8^{+} \mathrm{T}$ cells correlated with response to PD-1 blockade. Interestingly, the PD-1 blockade responder cells $\left(\mathrm{PD}-1^{+} \mathrm{CXCR} 5^{+}\right.$) were predominately 
A

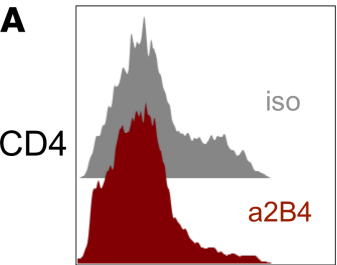

PD-1

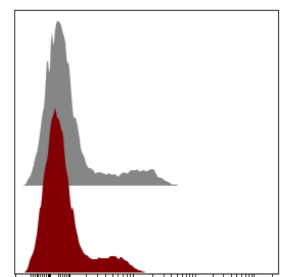

TIGIT

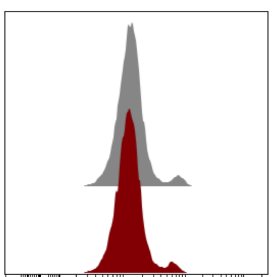

BTLA

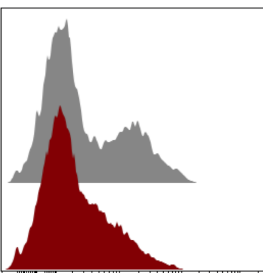

CTLA-4
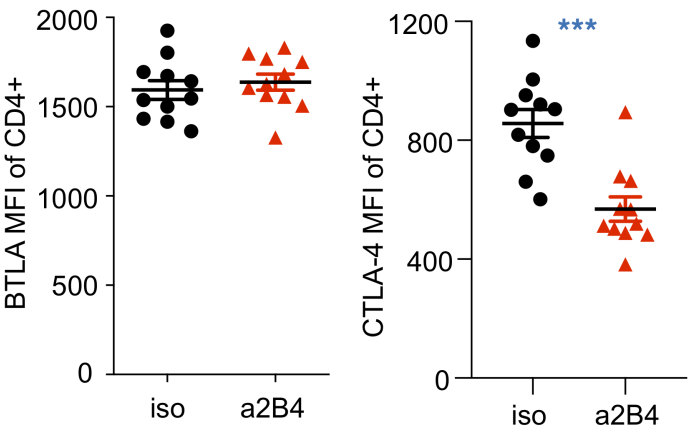

B

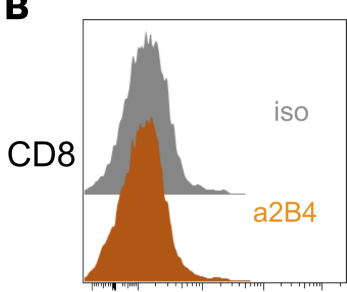

PD-1

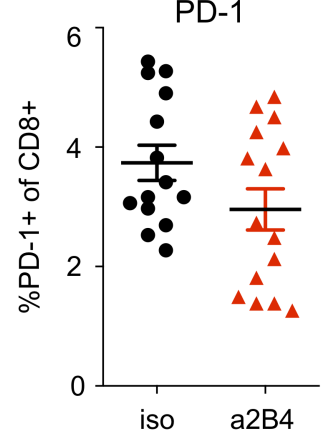

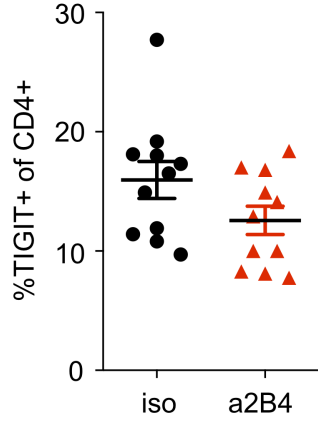

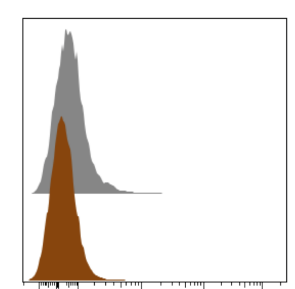

TIGIT

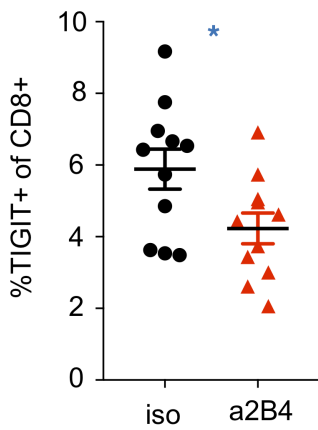

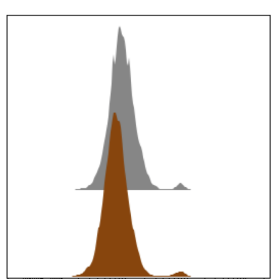

BTLA

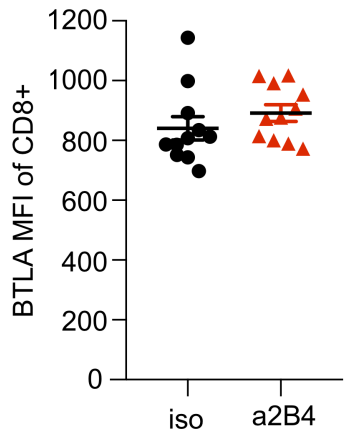

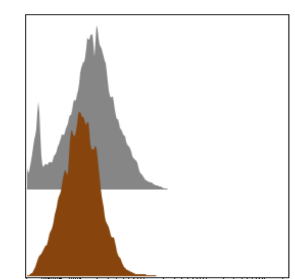

CTLA-4

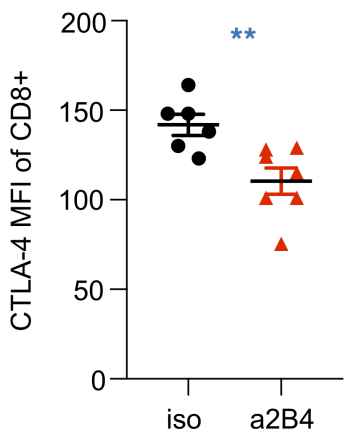

Figure 9. T cells from cancer septic animal exhibit reduction of coinhibitory receptors after anti-2B4 treatment. Splenic T cells were harvested and the expression of coinhibitory receptors was assessed 24 hours after cecal ligation and puncture (CLP) in the presence or absence of 2B4 blockade. (A) Summary plots of coinhibitory receptors on total CD4 ${ }^{+} T$ cells. (B) Summary plots of coinhibitory receptors on total CD8 ${ }^{+} \mathrm{T}$ cells. $n=6-14 /$ group. The 2 -tailed Student's $t$ test was performed. ${ }^{*} P<0.05,{ }^{* *} P<0.01$.

2B4- and had a transcriptional profile characteristic of memory precursor $\mathrm{T}$ cells with the expression of $\mathrm{Bcl}-$ 6, TCF-1. In contrast, the PD-1+2B4+ cells were mostly CXCR5- with more terminal differentiation profiles and were incapable of responding to the PD-1 blockade. In our study, we found decreased frequencies of CXCR5 ${ }^{+}$cells and increased frequencies of $2 \mathrm{~B} 4^{+}$cells among PD- $1^{+} \mathrm{T}$ cells in cancer septic animals (Figure $3)$, suggesting the composition of the $\mathrm{PD}-1^{+}$population during sepsis shifted toward becoming more terminally differentiated. Moreover, these data were also consistent with the SPADE and CITRUS data (Figure 4 and Figure 5) that $\mathrm{PD}-1 \mathrm{hi}_{2} \mathrm{~B} 4 \mathrm{hi}$ was identified in cancer septic animals compared with $\mathrm{PH}$ septic animals. Taken together, we have shown that both the loss of the quality and quantity of PD- $1^{+}$cells may have lead to the lack of efficacy of PD-1 blockade in cancer septic animals.

SPADE and CITRUS analysis revealed that 2B4 and PD-1-expressing CD8 ${ }^{+} \mathrm{T}$ cells constituted different populations during sepsis, suggesting that these coinhibitory receptors may have different roles during sepsis. These data were similar with our previously published data on $\mathrm{CD} 4^{+} \mathrm{T}$ cells in which we found 1 population was $2 \mathrm{~B} 4^{\text {hi }} \mathrm{PD}-1^{10} \mathrm{CD} 4^{+} \mathrm{T}$ cells and $2 \mathrm{~B} 4^{1 \mathrm{lo}} \mathrm{PD}-1^{\mathrm{hi}} \mathrm{CD} 4^{+} \mathrm{T}$ cells were elevated in cancer septic 
A

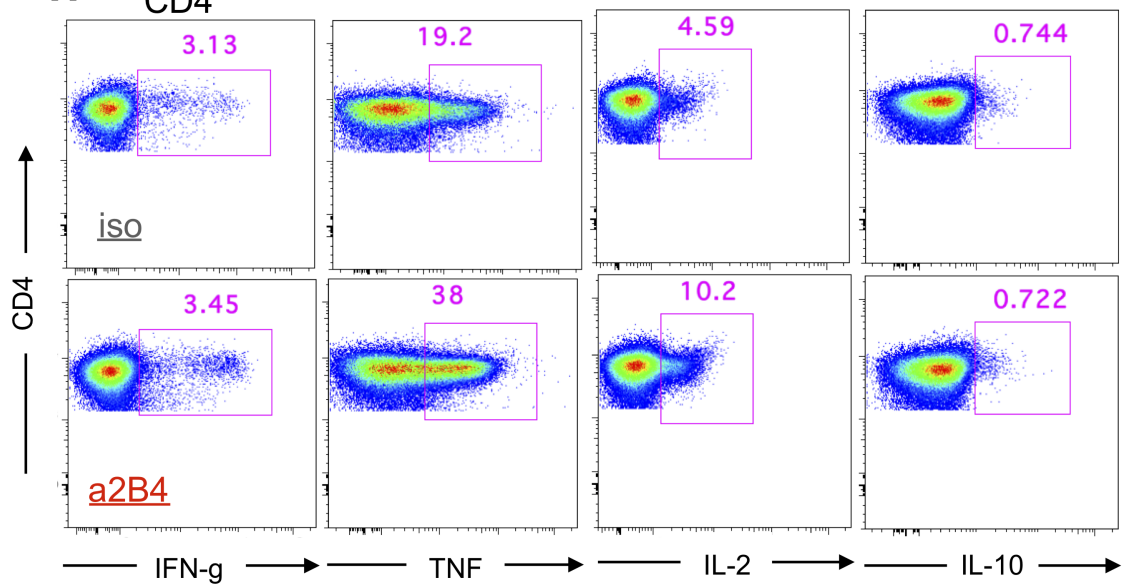

B $\quad$ CD8

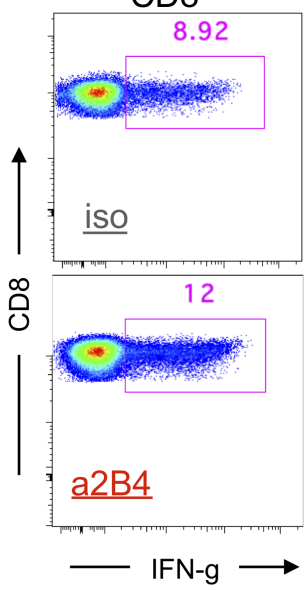

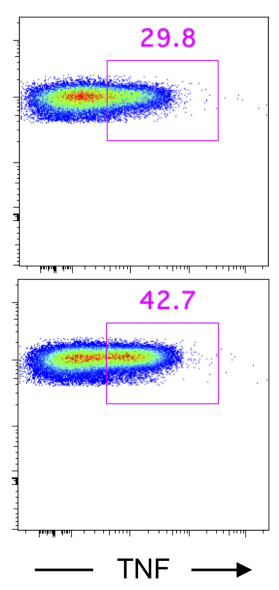

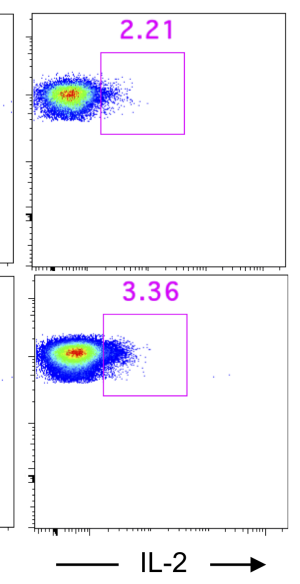

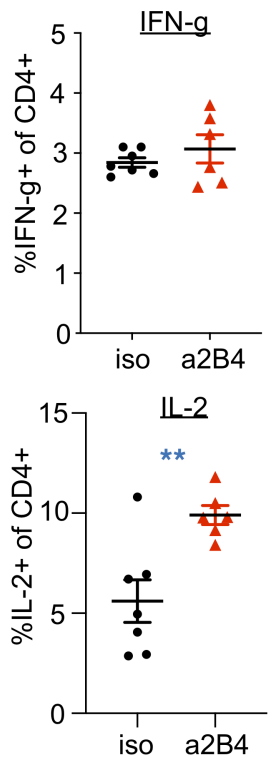
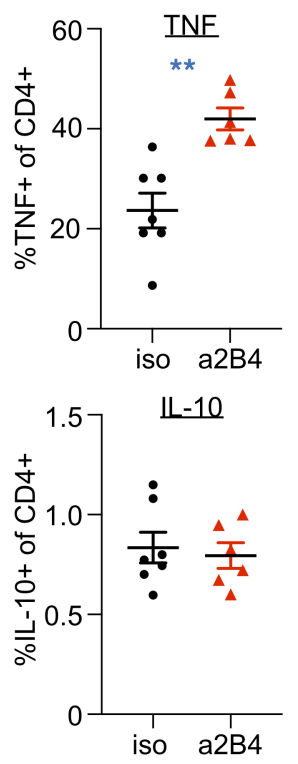

Figure 10. 2B4 blockade in cancer septic animals increases T cell cytokine secretion during sepsis. Cancer septic animals were subjected to cecal ligation and puncture (CLP), received 1 dose of anti-2B4 mAb or isotype control after surgery, and were sacrificed at 24 hours after CLP. Splenocytes were harvested and stimulated ex vivo with PMA and ionomycin for 4 hours, then were assessed for IFN- $\gamma$, TNF, IL-2, and IL-10 production via intracellular cytokine staining. (A) Representative flow plots and summary figures for CD4+ T cell cytokine staining. (B) Representative flow plots and summary figures for CD8 ${ }^{+}$T cell cytokine staining. The 2-tailed Student's $t$ test was performed. ${ }^{*} P<0.05,{ }^{*} P<0.01$. Data shown ( $n=6-7$ mice/ group) are representative of 2 independent experiments with a total of $n=12$ mice/group.

animals (37). Similar populations were identified in both $\mathrm{CD}^{+}$and $\mathrm{CD} 8^{+} \mathrm{T}$ cells (Supplemental Figure 4 ), strengthening the idea that $2 \mathrm{~B} 4$ and $\mathrm{PD}-1$ played distinct functions during cancer sepsis. We previously showed that 2B4-deficient animals were protected from CLP and blockade of 2B4 on PH WT animals improved sepsis survival. We found that the survival benefits of 2B4-deficient animals were derived from increased IFN- $\gamma$ secretion on $\mathrm{CD}^{+} \mathrm{T}$ cells (25); however, the mechanisms underlying the $2 \mathrm{~B} 4$ blockade remain unclear. Here we demonstrated anti-2B4 treatment increased CD48 expression and decreased PD-1 and CTLA-4 expression on T cells. CD48 is the ligand for CD2, which provides another T cell costimulatory signal. We speculated that during sepsis, $\mathrm{T}$ cells may rely more on costimulatory signals downstream of CD2 because of a significant decline in CD28 expression (Figure 8, A and B). Blockade of $2 \mathrm{~B} 4$ not only reduced the inhibitory signaling to $\mathrm{T}$ cells but also decreased competition for $\mathrm{CD} 48$, potentially allowing increased CD2 costimulatory signaling and cytokine secretion effector function. These possibilities warrant further investigation. Interestingly, PD-1 blockade does not affect 2B4 expression on T cells (data not shown). These results strengthen the idea that 2B4 and PD-1 play distinct and unique roles in the pathophysiology of sepsis in animals with cancer. However, we were not able to rule out a role for other 2B4-expressing immune cells, such as NK cells and gamma-delta T cells in our model. 
A

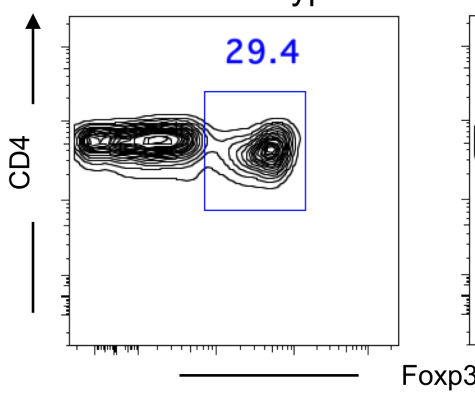

C

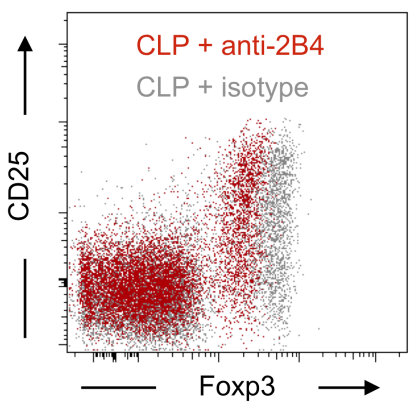

E

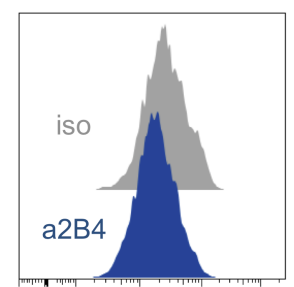

GITR

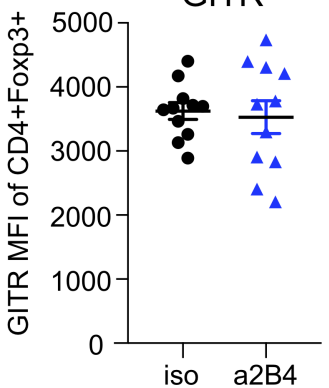

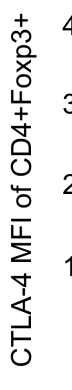

CLP + anti-2B4
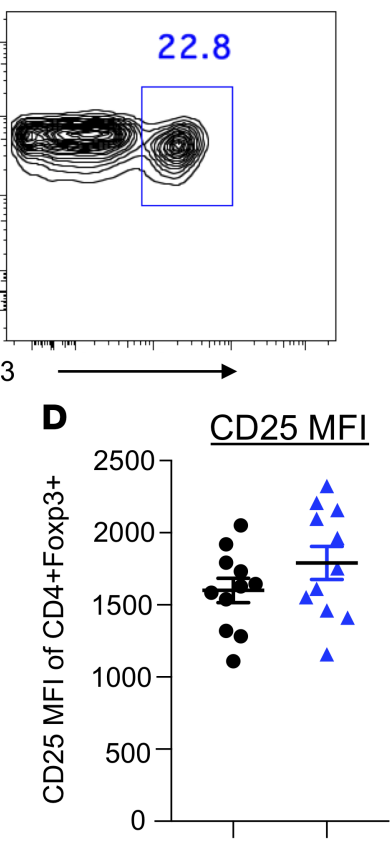

iso

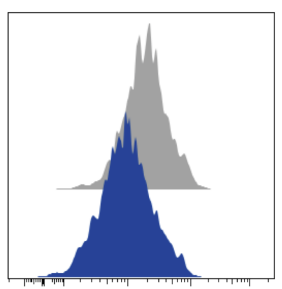

CTLA-4
***
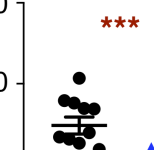

$2000-$

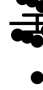

$\therefore \frac{4}{4-1}$

1000

0

iso a2B4
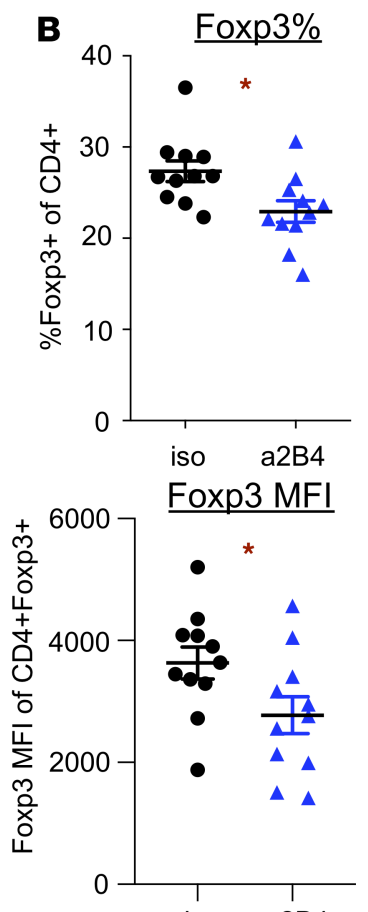

$\mathbf{F}$
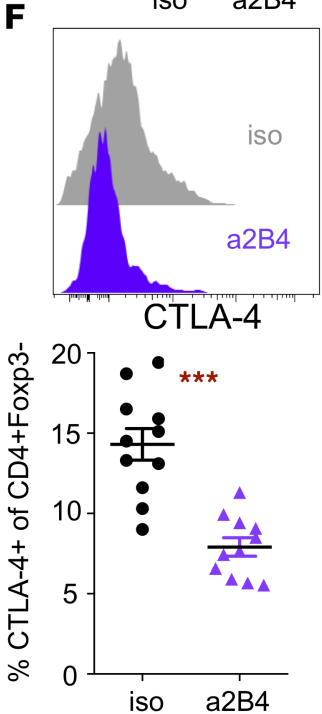

Figure 11. 2B4 blockade in cancer septic animals reduces frequency of and CTLA-4 expression on FoxP3+ cells during sepsis. Cancer septic animals were subjected to cecal ligation and puncture (CLP), received 1 dose of anti2B4 mAb or isotype control after surgery, and were sacrificed at 24 hours after CLP. (A) Representative flow plots depicting frequencies of $\mathrm{FoxP}^{+} \mathrm{T}$ cells in anti-2B4-treated cancer septic animals. The cells were gated on $\mathrm{CD3^{+ }} \mathrm{CD4}^{+}$ T cells. (B) Overlay dot plot of total $C D 3^{+} C D 4^{+} T$ cells isolated from anti-2B4-treated or isotype-treated cancer septic animals. (C) Representative flow figure depicting percentage of FoxP3 ${ }^{+} \mathrm{T}$ cells among total CD4 ${ }^{+} \mathrm{T}$ cells. (D) Summary figure depicting CD25 MFI and FoxP3 MFI of CD4 $4^{+} \mathrm{FoxP3}^{+} \mathrm{T}$ cells. (E) Representative flow plots and summary data depicting GITR and CTLA-4 expression on $\mathrm{CD}^{+} \mathrm{FoxP3}^{+} \mathrm{T}$ cells isolated from control versus anti-2B4-treated cancer septic animals. (F) Representative flow plots and summary data depicting CTLA-4 expression on CD4+ ${ }^{+}$oxP3- $\mathrm{T}$ cells isolated from control versus anti-2B4-treated cancer septic animals. $n=11$ in each group. The 2-tailed Student's $t$ test was performed. ${ }^{*} P<0.05,{ }^{* *} P<0.001$.

One of the limitations of this study was that the result might be tumor-type specific or sepsis model-specific. LLC1 is the Lewis lung carcinoma cell line, which is highly tumorigenic and widely used as a model of metastasis and to evaluate the efficacy of therapeutic agents (39). We chose LLC1 as our tumor model for several reasons: first, lung cancer represents the most common type of cancer in cancer septic patients. Second, PD-1 blockade therapy has previously been shown to not reduce LLC1 tumor size in the 
mouse model (31); this was also true in our system (data not shown), allowing us to isolate the impact of PD-1 blockade on cancer sepsis survival.

This study emphasized the complexity and contradiction in preexisting host comorbidity with sepsis. Despite the negative results using PD-1 blockade in our model, targeting immune dysfunction via 2B4 blockade remains a promising direction for sepsis with cancer comorbidity. Our results should be repeated using different types of tumors and other models of sepsis to determine the generalizability of the impact of PD-1 or 2B4 blockade in cancer septic hosts. In the future, combination therapy targeting multiple checkpoints could represent promising strategies for successful immune therapy in the setting of cancer sepsis. For example, we observed that CD4 ${ }^{+} \mathrm{PD}-1^{+} \mathrm{T}$ cells in animals with cancer upregulated CD127 (IL-7 receptor) during sepsis (not shown); therefore, combined PD-1 blockade IL-7 administration could be investigated (40). In addition, clinical factors or potential biomarkers should be considered carefully in the criteria for future clinical sepsis trials aimed at checkpoint therapy. The septic patients with cancer comorbidity should be excluded from general septic patient populations and precision medicine strategies should be developed to more optimally improve sepsis-induced immune dysregulation in the setting of preexisting malignancy.

\section{Methods}

Animals and cancer model. C57BL/6 mice with aged from 8-12 weeks old; both male and female were purchased from Jackson Laboratory. All animals were housed and maintained by following Emory IACUC guidelines. For cancer animal model, 500,000 murine lung cancer cell line LLC1 (ATCC, CRL-1642) cells were implanted in the right thigh via s.c. injection. After 3 weeks, the cancer-bearing animals were randomly divided into different groups for experiments. LLC1 was cultured in DMEM (Sigma-Aldrich) with 10\% FBS, Penicillin-Streptomycin (100 U/mL, Thermo Fisher Scientific) and HEPES (0.01M, Sigma-Aldrich). The LLC1 cancer cell culture and cryopreservation methods were followed by ATCC guidelines.

Cecal ligation and puncture. Polymicrobial animal sepsis model, CLP was performed to induce sepsis on cancer animals. Following the method of Baker et al. (41), the cecum was ligated and punctured twice with a 25-gauge needle. After surgery, septic animals received $1 \mathrm{~mL}$ of subcutaneous saline at 0 hours, and 4 doses of antibiotics ( $50 \mathrm{mg} / \mathrm{kg}$ ceftriaxone and $35 \mathrm{mg} / \mathrm{kg}$ metronidazole, Sigma-Aldrich) at 0, 12, 24, and 36 hours. Sham surgery was performed as a control group. Animals were provided with pain medication ( $0.1 \mathrm{mg} / \mathrm{kg}$ buprenex, McKesson Medical) to minimize pain before the surgery.

Antibody blockade. For anti-PD-1 treatment, animals were treated with 2 different dosing strategies. The delayed administration of anti-PD-1 was followed by the method of Brahmamdam et al. (19) $250 \mu \mathrm{g}$ of PD-1 antagonistic mAb (clone RMP1-14, isotype Rat IgG2a, , Bio X Cell, Inc.) or isotype control antibody was administered by i.p. injection on days 1 and 2 after CLP. An alternative method of anti-PD-1 blockade was performed by administration of $250 \mu \mathrm{g}$ anti-PD-1 mAb (clone 29F.1A12, isotype Rat IgG2a, Bio X Cell) on days 0, 2, 4, and 6 after CLP. For anti-2B4 treatment, animals received anti-2B4 mAb (clone 2B4, 250 mg per dose, i.p. injection, anti-2B4 was a gift of Vinay Kumar, University of Chicago, Chicago, Illinois, USA) on days $0,2,4$, and 6 after CLP.

Flow cytometry antibodies and reagents. Antibodies (clone) for flow cytometry were purchased from the following companies: BioLegend: CD2 (RM2-5), CD8 (53-6.7), CD28 (E18), CD44 (IM7), CD127 (A7R34), CD62L (MEL-14), CD48 (HM48-1), Tim-3 (RMT3-23), PD-1 (29F1A12), ICOS (C398.4A), CTLA-4 (UC10-4B9), LAG-3 (C987W), TIGIT (IG9), CXCR5 (L138D7), PD-L1 (10F.9G2), IFN- $\gamma$ (XMG1.2), and TNF (MP6-XT22); eBioscience Thermo Fisher Scientific: 2B4 (eBio244F4), BTLA (6F7), Foxp3 (FJK16s), and IL-10 (JES5-16E3); BD Pharmingen: CD3 (500A2), CD4 (RM4-5), and IL-2 (JES6-5H4); and Cell Signalling Technology: Bcl-xL (54H6). For Foxp3, Bcl-XL, and total CTLA4 staining, splenocytes were stained with surface markers and then fixed and permeabilized by eBioscience Foxp3/Transcription Factor Staining Buffer Set (Catalog: 00-5523-00). CellEvent Caspase-3/7 Green Flow Cytometry Assay Kit (Catalog: C10427) was used for activated Caspase 3/7 staining. Absolute cell counts were obtained by using CountBright Absolute Counting Beads (Catalog: C36950). For intracellular cytokine staining, splenocytes were incubated with $30 \mathrm{ng} / \mathrm{mL}$ PMA and $1 \mathrm{nM}$ ionomycin in the presence of GolgiStop for 4 hours at $37^{\circ} \mathrm{C}$. Flow analysis was performed on FlowJo and Cytobank.

SPADE and CITRUS analysis. PH animals and those with cancer were subjected to CLP, and 24 hours after CLP, splenocytes were collected and stained for CD3, CD4, CD8, CD44, BTLA, PD-1, 2B4, and LAG-3. Files containing only $\mathrm{CD}^{+} \mathrm{CD}^{+} \mathrm{T}$ cells were exported from traditional flow data (FlowJo) and uploaded to Cytobank (Cytobank.org) for automated analysis. Two different algorithms were performed: 
SPADE (Spanning-tree Progression Analysis of Density-normalized Events) and CITRUS (cluster identification, characterization, and regression). For SPADE analysis, PH septic animals were set as baseline and cancer septic animals were defined as the fold changed group. The node size was defined as 50 nodes to have at least 100 cells in 1 node. The SPADE trees were generated by the parameter "percent total ratio log" to visualize the difference between groups. For SPADE statistic, the percent of total CD8 ${ }^{+}$cells in each node was compared by multiple $t$ tests with adjusted $P$ values (FDR $=1 \%$ ), and significantly different nodes were grouped into clusters based on the phenotypically similarity. For CITRUS, the PAMR association model and minimal FDR were selected. The CITRUS program generated the tree-like figures, phenotype figures, defined clusters, and statistics automatically without manual input.

Statistics. The 2-tailed Student's $t$ test, multiple $t$ test, 1-way ANOVA, and log-rank test were used. The outlier is removed by performing Grubbs' test. Data are presented as mean \pm SEM. $\left({ }^{*} P<0.05 ;{ }^{*} P<0.01\right.$; *** $P<0.001)$

Study approval. Animal studies were reviewed and approved by Emory Institutional Animal Care and Use Committee, Atlanta, Georgia, USA (protocol number DAR-2003199-ELEMNT-N).

\section{Author contributions}

CWC, CMC, and MLF designed and directed the project; CWC performed and analyzed all experiments; WZ, MX, and JX performed experiments; CWC wrote the manuscript with support from CMC and MLF. All authors discussed the results and edited the final manuscript.

\section{Acknowledgements}

The authors wish to thank Dr. Jennifer M. Robertson for assistance with flow cytometry and Dr. Zhe Liang for technical assistance. This work was funded by R01s GM104323 and GM113228 to MLF and CMC.

Address correspondence to: Craig M. Coopersmith or Mandy L. Ford, 101 Woodruff Circle, Suite 5105 Woodruff Memorial Research Building. Emory University, Atlanta, Georgia 30322, USA. Phone: 404727-2900; Email: cmcoop3@emory.edu (C.M. Coopersmith); mandy.ford@emory.edu (M.L. Ford).

1. Rhee C, et al. Incidence and trends of sepsis in US hospitals using clinical vs claims data, 2009-2014. JAMA. 2017;318(13):1241-1249.

2. Angus DC, Linde-Zwirble WT, Lidicker J, Clermont G, Carcillo J, Pinsky MR. Epidemiology of severe sepsis in the United States: analysis of incidence, outcome, and associated costs of care. Crit Care Med. 2001;29(7):1303-1310.

3. Singer M, et al. The Third International Consensus Definitions for Sepsis and Septic Shock (Sepsis-3). JAMA. 2016;315(8):801-810.

4. Melamed A, Sorvillo FJ. The burden of sepsis-associated mortality in the United States from 1999 to 2005: an analysis of multiple-cause-of-death data. Crit Care. 2009;13(1):R28.

5. Williams MD, et al. Hospitalized cancer patients with severe sepsis: analysis of incidence, mortality, and associated costs of care. Crit Care. 2004;8(5):R291-R298.

6. Danai PA, Moss M, Mannino DM, Martin GS. The epidemiology of sepsis in patients with malignancy. Chest. 2006;129(6):1432-1440.

7. Abou Dagher G, et al. Are patients with cancer with sepsis and bacteraemia at a higher risk of mortality? A retrospective chart review of patients presenting to a tertiary care centre in Lebanon. BMJ Open. 2017;7(3):e013502.

8. Soares $\mathrm{M}$, et al. Characteristics and outcomes of patients with cancer requiring admission to intensive care units: a prospective multicenter study. Crit Care Med. 2010;38(1):9-15.

9. Rosolem MM, et al. Critically ill patients with cancer and sepsis: clinical course and prognostic factors. J Crit Care. 2012;27(3):301-307.

10. Chen DS, Mellman I. Oncology meets immunology: the cancer-immunity cycle. Immunity. 2013;39(1):1-10.

11. Schreiber RD, Old LJ, Smyth MJ. Cancer immunoediting: integrating immunity's roles in cancer suppression and promotion. Science. 2011;331(6024):1565-1570.

12. Thommen DS, Schumacher TN. T cell dysfunction in cancer. Cancer Cell. 2018;33(4):547-562.

13. Bodey GP. Infection in cancer patients. A continuing association. Am J Med. 1986;81(1A):11-26.

14. Mittal R, et al. Murine lung cancer induces generalized T-cell exhaustion. J Surg Res. 2015;195(2):541-549.

15. van der Burg SH, Arens R, Ossendorp F, van Hall T, Melief CJ. Vaccines for established cancer: overcoming the challenges posed by immune evasion. Nat Rev Cancer. 2016;16(4):219-233.

16. Hotchkiss RS, Monneret G, Payen D. Immunosuppression in sepsis: a novel understanding of the disorder and a new therapeutic approach. Lancet Infect Dis. 2013;13(3):260-268.

17. Boomer JS, et al. Immunosuppression in patients who die of sepsis and multiple organ failure. JAMA. 2011;306(23):2594-2605

18. Hotchkiss RS, et al. Immune checkpoint inhibition in sepsis: a phase $1 \mathrm{~b}$ randomized, placebo-controlled, single ascending dose study of Antiprogrammed Cell Death-Ligand 1 Antibody (BMS-936559). Crit Care Med. 2019;47(5):632-642. 
19. Brahmamdam P, Inoue S, Unsinger J, Chang KC, McDunn JE, Hotchkiss RS. Delayed administration of anti-PD-1 antibody reverses immune dysfunction and improves survival during sepsis. J Leukoc Biol. 2010;88(2):233-240.

20. Zhang Y, et al. PD-L1 blockade improves survival in experimental sepsis by inhibiting lymphocyte apoptosis and reversing monocyte dysfunction. Crit Care. 2010;14(6):R220.

21. Chang KC, et al. Blockade of the negative co-stimulatory molecules PD-1 and CTLA-4 improves survival in primary and secondary fungal sepsis. Crit Care. 2013;17(3):R85

22. Inoue S, Bo L, Bian J, Unsinger J, Chang K, Hotchkiss RS. Dose-dependent effect of anti-CTLA-4 on survival in sepsis. Shock. 2011;36(1):38-44

23. Cheng T, Bai J, Chung CS, Chen Y, Biron BM, Ayala A. Enhanced innate inflammation induced by anti-BTLA antibody in dual insult model of hemorrhagic shock/sepsis. Shock. 2016;45(1):40-49.

24. Shubin NJ, Chung CS, Heffernan DS, Irwin LR, Monaghan SF, Ayala A. BTLA expression contributes to septic morbidity and mortality by inducing innate inflammatory cell dysfunction. J Leukoc Biol. 2012;92(3):593-603.

25. Chen CW, et al. Cutting Edge: 2B4-mediated coinhibition of $\mathrm{CD}^{+} \mathrm{T}$ cells underlies mortality in experimental sepsis. J Immunol. 2017;199(6):1961-1966

26. Kamphorst AO, et al. Proliferation of PD-1+ CD8 T cells in peripheral blood after PD-1-targeted therapy in lung cancer patients. Proc Natl Acad Sci U S A. 2017;114(19):4993-4998.

27. Kamphorst AO, et al. Rescue of exhausted CD8 T cells by PD-1-targeted therapies is CD28-dependent. Science. 2017;355(6332):1423-1427.

28. Im SJ, et al. Defining CD8 ${ }^{+} \mathrm{T}$ cells that provide the proliferative burst after PD-1 therapy. Nature. 2016;537(7620):417-421

29. Bai M, Zheng Y, Liu H, Su B, Zhan Y, He H. CXCR5 ${ }^{+} \mathrm{CD}^{+}$T cells potently infiltrate pancreatic tumors and present high functionality. Exp Cell Res. 2017;361(1):39-45.

30. Brummelman J, et al. High-dimensional single cell analysis identifies stem-like cytotoxic $\mathrm{CD}^{+} \mathrm{T}$ cells infiltrating human tumors. J Exp Med. 2018;215(10):2520-2535.

31. Lin H, et al. Host expression of PD-L1 determines efficacy of PD-L1 pathway blockade-mediated tumor regression. $J$ Clin Invest. 2018;128(2):805-815

32. Tang H, et al. PD-L1 on host cells is essential for PD-L1 blockade-mediated tumor regression. J Clin Invest. 2018;128(2):580-588.

33. Hotchkiss RS, et al. Caspase inhibitors improve survival in sepsis: a critical role of the lymphocyte. Nat Immunol. 2000;1(6):496-501.

34. Hotchkiss RS, et al. Prevention of lymphocyte cell death in sepsis improves survival in mice. Proc Natl Acad Sci U S A. 1999;96(25):14541-14546.

35. Wesche-Soldato DE, Chung CS, Lomas-Neira J, Doughty LA, Gregory SH, Ayala A. In vivo delivery of caspase-8 or Fas siRNA improves the survival of septic mice. Blood. 2005;106(7):2295-2301.

36. Fox AC, et al. Prevention of lymphocyte apoptosis in septic mice with cancer increases mortality. J Immunol. 2011;187(4):1950-1956.

37. Xie J, Robertson JM, Chen CW, Zhang W, Coopersmith CM, Ford ML. Pre-existing malignancy results in increased prevalence of distinct populations of CD4+ T cells during sepsis. PLoS One. 2018;13(1):e0191065.

38. Mittal R, et al. Phenotypic T cell exhaustion in a murine model of bacterial infection in the setting of pre-existing malignancy. PLoS One. 2014;9(5):e93523

39. Zhao L, et al. Late-stage tumors induce anemia and immunosuppressive extramedullary erythroid progenitor cells. Nat Med 2018;24(10):1536-1544.

40. Francois B, et al. Interleukin-7 restores lymphocytes in septic shock: the IRIS-7 randomized clinical trial. JCI Insight. 2018;3(5):98960.

41. Baker CC, Chaudry IH, Gaines HO, Baue AE. Evaluation of factors affecting mortality rate after sepsis in a murine cecal ligation and puncture model. Surgery. 1983;94(2):331-335. 